\title{
Computational reconstruction of mental representations using human behavior
}

\author{
Laurent Caplette $^{1, *} \&$ Nicholas B. Turk-Browne $e^{1,2}$ \\ ${ }^{1}$ Department of Psychology, Yale University, New Haven, CT, U.S.A. \\ ${ }^{2} \mathrm{Wu}$ Tsai Institute, Yale University, New Haven, CT, U.S.A.
}

*Corresponding author

Email: laurent.caplette@yale.edu

Address: Department of Psychology, Yale University, 2 Hillhouse Avenue, New Haven, CT, 06520, U.S.A. 


\begin{abstract}
Revealing the contents of mental representations is a longstanding goal of cognitive science. However, there is currently no general framework for providing direct access to representations of high-level visual concepts. We asked participants to indicate what they perceived in images made of random visual features in a deep neural network. We then inferred a mapping between the semantic features of their responses and the visual features of the images. This allowed us to reconstruct the mental representation of virtually any common visual concept, both those reported and others extrapolated from the same semantic space. We successfully validated 270 of these reconstructions in separate participants. The visual-semantic mapping uncovered with our method further generalized to new stimuli and a new task. Finally, it allowed us to reconstruct representations of individual observers and of a neural network. This framework enables a large-scale investigation of conceptual representations.
\end{abstract}


Revealing the information contents of memory is central to elucidating the mechanisms underlying cognition. For example, categorization requires the match of stimulus features to a memorized representation, prediction relies on the transfer of memorized information to sensory areas, and learning updates memorized representations to incorporate new information. Knowing what information is represented is a necessary part of understanding at an algorithmic level how the brain performs a particular task: one cannot know how the mind works without relating specific behaviors to specific stimuli ${ }^{1-3}$. Accessing the content of mental representations is necessary to understand the human mind because it allows us to link sensory features to behaviors. Such knowledge can in turn inform models of human behavior that make specific predictions. For example, understanding which features are part of someone's mental representation of the concept "trustworthy" helps us to predict how they will act in social interactions with different individuals. Characterizing mental representations is not easy, however, as they are not directly observable.

One way to understand the behavior of a black box like this is by probing it with noise ${ }^{4}$. This idea is the basis of the reverse correlation paradigm ${ }^{5-7}$. In the purest form of this paradigm, participants are shown random noise (e.g., pixel noise) on every trial but told that there is a hidden signal on half of the trials (e.g., the letter "s") and that they must respond when they think the signal was shown ${ }^{8}$. Averaging the stimuli associated with these "superstitious" detections reveals the mental representation of that signal, unbiased by external input. This approach has been used to recover representations of letters, facial expressions, and 3D patterns ${ }^{8-11}$. Findings from these and related methods informed and validated models and theories of letter identification, universality of facial expressions, and trustworthiness judgments, among other aspects of cognition ${ }^{10-13}$. One prominent disadvantage of this method however is that it typically requires thousands of trials per observer and is limited to artificial or simple target signals that always have the same pixel representation.

Natural visual categories are defined by abstract features invariant under many linear and nonlinear image transformations, rendering the traditional pixel-based reverse correlation approach inappropriate for capturing their representations. Instead, random sampling of features learned by intermediate layers of convolutional neural networks (CNNs) - "CNN features", for short - might be more appropriate for this goal. CNNs are deep neural networks that can be trained on thousands or millions of natural images to learn abstract features that allow them, among other tasks, to categorize objects with a high accuracy ${ }^{14}$. Features from intermediate layers can correspond to object parts or complex textures that map to multiple representations in pixel space (e.g., multiple potential colors, shapes, and orientations) $)^{15-16}$. Of course, such $\mathrm{CNN}$ features have reduced expressiveness compared 
to pixels: whereas sampling pixels can allow the reconstruction of any image, sampling CNN features limits the space of possible reconstructions. At the same time, CNN features are likely closer to the features represented in the human brain, and so the subspace of images that can be reconstructed may be the one most relevant to capturing concepts from the natural world. These features resemble the features represented by mid- and high-level areas of visual cortex ${ }^{17-19}$. Recent neuroimaging experiments have exploited this finding to recover mental representations of natural images ${ }^{20-24}$ or to construct "superstimuli" that maximize the activity of a given brain region ${ }^{25-26}$. By relating CNN features and brain responses, new brain responses can be translated into vectors of CNN feature values (representing how much each CNN feature is associated with these brain responses), which can in turn be used to reconstruct images corresponding to the visual contents of the brain. Existing studies have followed this approach to reconstruct mental representations of stimuli currently available from the external environment. Recovering internally generated representations has proven more challenging (e.g., with imagined stimuli ${ }^{24,27)}$.

An additional drawback of the reverse correlation and brain-based reconstruction approaches is that they focus on small sets of experimenter-defined stimuli. A more general, ambitious, and potentially fruitful approach would be to recover a function mapping the labels of all natural visual categories to visual features (see also refs. ${ }^{28-29}$ ). Because both category labels and visual representations can be conceptualized as being positioned in continuous multidimensional spaces, such a mapping might be computable. Moreover, it might be feasible to recover it, or a good approximation, with a reasonable amount of data. Indeed, because multiple labels are semantically related or interchangeable, the semantic space of relevant category labels is relatively low-dimensional ${ }^{30-32}$. Similarly, because multiple images depict the same category, the space of relevant visual representations is also relatively low-dimensional ${ }^{33-36}$.

In this study, we aimed to recover and visualize the representations of an arbitrarily large number of complex natural concepts. We focussed on natural concepts because, to have a model of human behavior in the real world, one needs to uncover the representations of useful real-world categories. Most importantly, we aimed to uncover a powerful method that would allow us to reconstruct many representations because this is a step toward a general model of human behavior. Focussing on the representation of a single category would allow us to understand and predict behavior pertaining to that category but may not inform us about other categories. Accessing many representations could allow us to study qualitatively different and more general questions, such as the optimality of representations overall, their dimensionality, and how they cluster together, for example. 
Finally, we aimed to reconstruct representations in single individuals. A method that achieves this aim would allow us to study inter-individual differences in representations and their origins, for example by relating these differences to distinct developmental, social, or cultural factors.
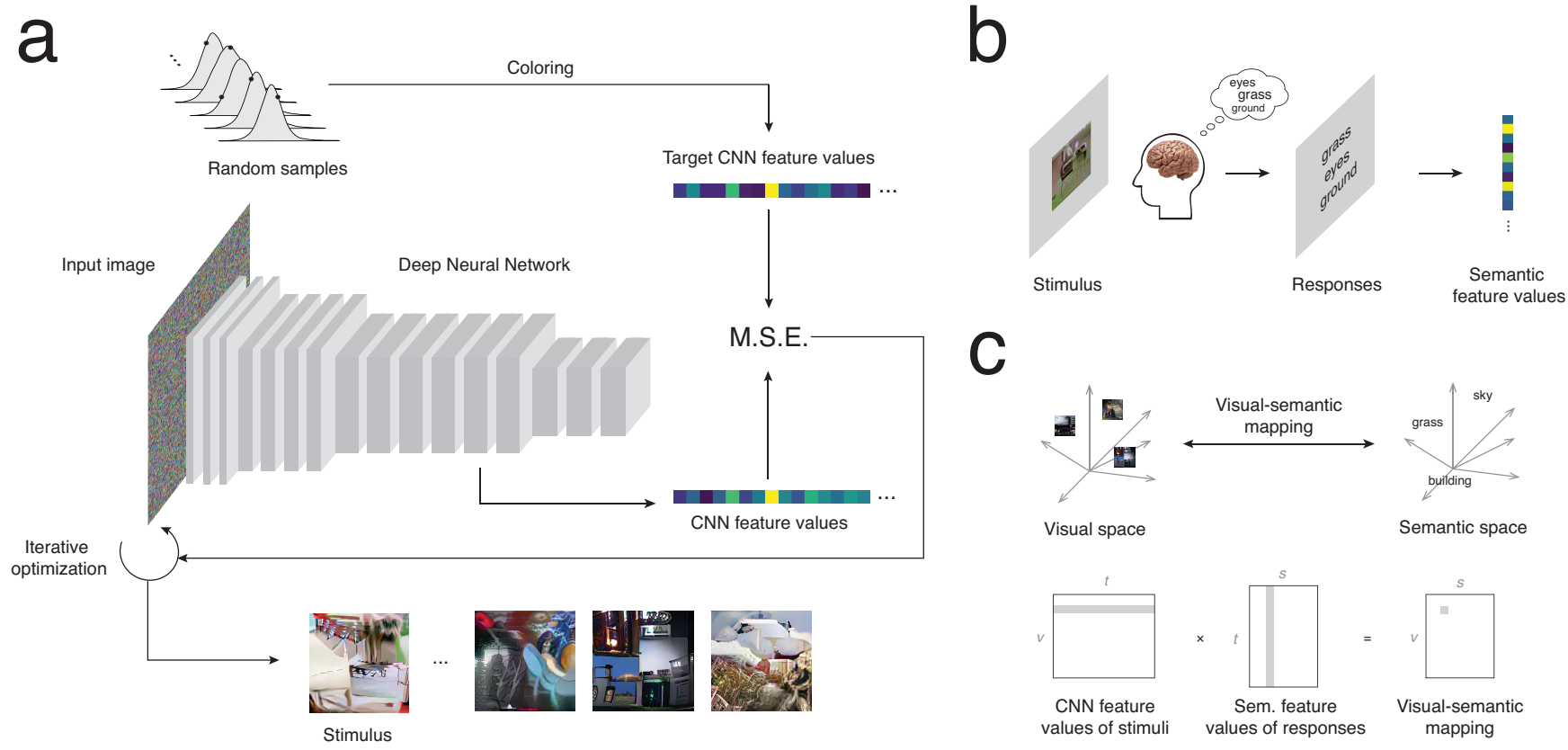

Figure 1. Experimental methods and analyses. A) Illustration of the stimulus synthesis procedure. Random samples are drawn from uncorrelated distributions. A coloring (inverse whitening) transform is applied to cast these samples to the original CNN feature space (these are the target $\mathrm{CNN}$ feature values). An image is iteratively optimized from noise so that its actual CNN feature values are similar to these target CNN feature values. The result is referred to as a "CNN-noise" stimulus (i.e., a stimulus whose CNN feature values are pseudorandom) and will be used in the experiment. Some stimulus examples are shown at the bottom of the panel. M.S.E. = Mean squared error. B) Experimental paradigm. On each trial, a CNN-noise stimulus is shown to a participant for $5 \mathrm{~s}$. The participant then writes 1-3 labels indicating what was perceived in the stimulus. These labels are transformed into a vector of semantic feature values summarizing the semantic content of the stimulus as perceived by the observer. C) Overview of the analyses. Visual representations and CNN-noise stimuli lie in a high-dimensional space of CNN features (visual space). Label responses lie in a high-dimensional space of semantic features (semantic space). We can infer a mapping between these spaces by taking the matrix product of the $v(\mathrm{CNN}$ visual features) $\times t$ (trials) matrix of stimuli and the $t \times s$ (semantic features) matrix of responses. The result is a $v \times s$ visual-semantic mapping matrix indicating how each CNN feature is related to each semantic feature. Gray shaded areas indicate an example CNN feature and its values across trials (left), an example semantic feature and its values across trials (middle), and their association inferred by the mapping (right).

We developed a method that generalizes reverse correlation in terms of both sensory inputs (stimuli) and behavioral outputs (labels). First, instead of sampling pixels, we pseudo-randomly sampled CNN features, i.e., the abstract features to which CNN channels respond. The result is a vector of $\mathrm{CNN}$ feature values, i.e., a vector of values associated with each feature. These serve as our target feature values for the creation of a stimulus: on each trial, we iteratively optimized an image so that its $\mathrm{CNN}$ feature values corresponded to these target feature values. (Note that we refer to this process as sampling CNN features for simplicity, even though we cannot directly sample them and must resort to an iterative optimization procedure; see Methods: $\mathrm{CNN}$-noise stimuli.) The final optimized image is a mix of random CNN features in which no object is clearly visible ("CNN-noise" stimulus; Figure 1a). Then, rather than focusing on one or a small set of predefined categories, we asked observers to 
indicate all categories they perceived in the stimuli, and we transformed their responses to semantic feature values using a pretrained word embedding (Figure 1b). Such an embedding maps words to features in a continuous multidimensional space according to their semantic content. This experimental paradigm allowed us to map the entire semantic space of category labels to the visual space of CNN intermediate layer features, and thus to retrieve the visual features associated with any label, even ones that were not supplied during the experiment. From the translated feature values for a given label, we could then synthesize an image approximating the concept's mental representation using a procedure like that used to synthesize stimuli. Our aim for these reconstructions was not to perfectly capture all features of a mental representation but rather to create visualizations that were distinguishable from each other and that were generalizable to other tasks. In sum, we developed a new way to quantify and visualize abstract representations of natural visual concepts in semantic memory.

\section{Results}

\section{Mapping visual features to semantic features}

Participants provided an average of 2.17 labels per stimulus. After corrections and removal of invalid responses, this corresponded to 2,578 unique words, of which 369 were provided at least 10 times each (Figure 2a). The most frequent words were "grass" (607 responses), "sky" (592), "tree" (450), "dog" (355) and "bird" (355). 
We transformed each word into semantic feature values using a pretrained word embedding, averaged these vectors of feature values into a single one per stimulus, and reduced the dimensionality of these average semantic feature values using principal components analysis (PCA). The resulting principal components (PCs) are reduced representations of the semantic content of the stimuli as perceived by the observers. We also used PCA to reduce the dimensionality of the vectors of CNN feature values associated with the stimuli. Finally, we inferred a linear visual-semantic mapping between the semantic PCs and the CNN PCs (Figure 1c). The result is a matrix indicating how much each CNN PC is related to each semantic PC (Figure $2 b)$. There were 67 significant associations $(\mathrm{Z}<$ -4.76 or $\mathrm{Z}>4.75 ; \mathrm{p}<.05$, two-sided, FWER-corrected): 21 unique CNN PCs were positively and negatively associated to 14 unique semantic PCs. The most important semantic and CNN PCs, along with their associations, are visualized in Figure 2c. For example, a semantic PC associated with nature concepts (PC \#2) is strongly correlated with CNN PCs that partly code for grass-like and water-like textures (PCs \#1, \#4 and \#7). Similarly, a semantic PC associated with humans/animals (PC \#3) is

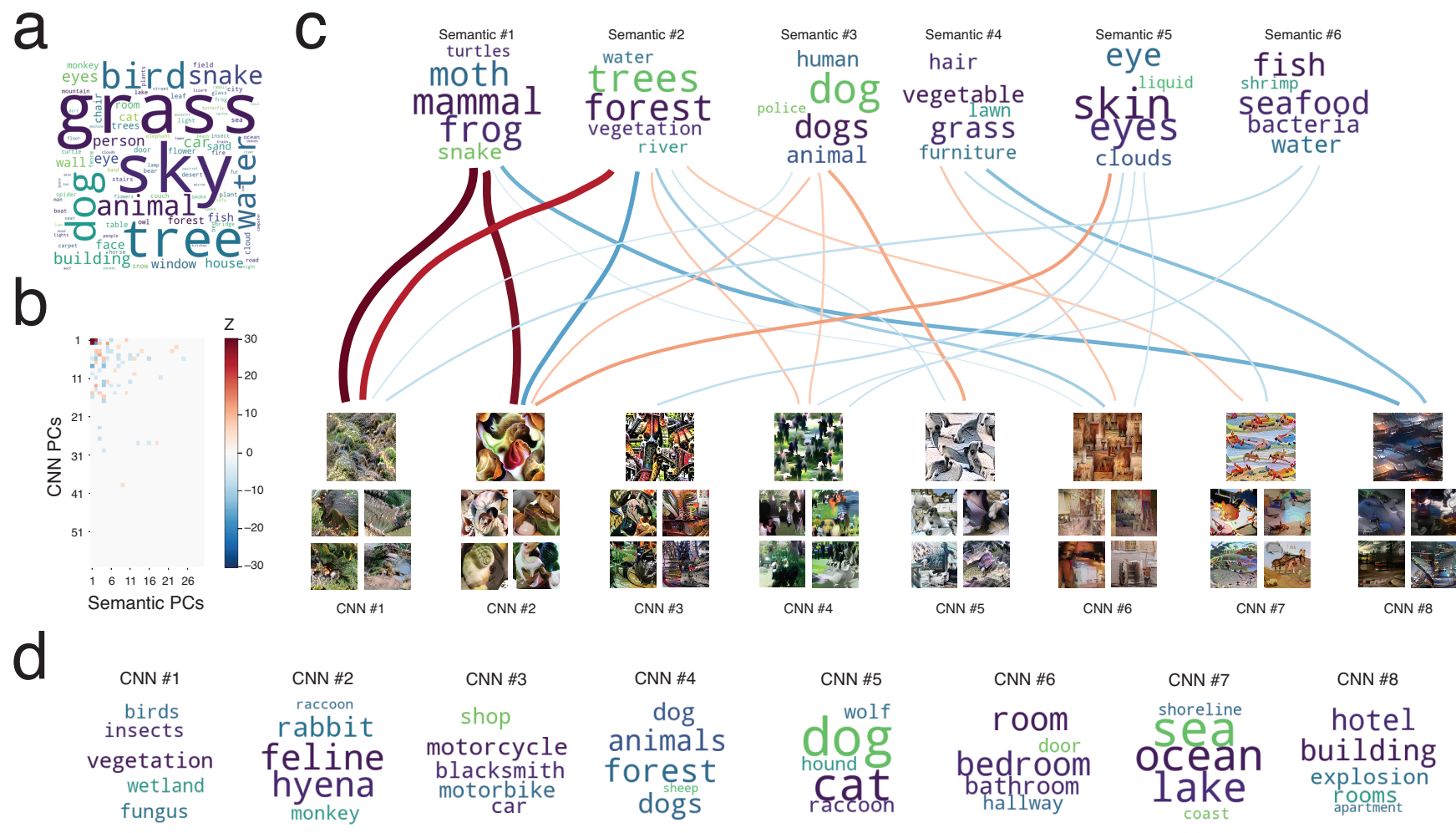

Figure 2. Relating visual and semantic features. A) The 100 most common responses provided by participants. The size of the word is approximately proportional to its frequency. B) Illustration of the significant associations between the first $60 \mathrm{CNN}$ principal components (PCs) and the first 30 semantic PCs ( $<<0.05$, two-sided, FWER-corrected). C) Detail of the significant associations between the first 6 semantic PCs and the first 8 CNN PCs. The color of the curves represents the strength of the association (see scale in panel B) and the thickness is proportional to its absolute value. Semantic PCs are summarized by the 5 words provided in the experiment that loaded most strongly (word size approximately proportional to closeness). CNN PCs are summarized by both a synthesized image maximizing the value of that PC (top) and the 4 stimuli that loaded most strongly (bottom). D) Summary of the semantic content associated with the top 8 CNN PCs. The 5 words that corresponded most closely to the semantic feature values associated with each CNN PC are shown (word size approximately proportional to closeness). 
related to CNN PCs seemingly representing skin textures, fur textures, and animal faces (PCs \#2, \#4 and \#5). Our mapping further allows us to uncover and visualize the words most associated with a given CNN PC, improving the interpretation of features (Figure 2d). This specific visualization method could be applied to units of different neural networks to aid explainability ${ }^{37}$ and reduce observer bias.

\section{Reconstructing mental representations of visual concepts}

Using the visual-semantic mapping we inferred, we can now retrieve the CNN feature values associated with any concept. Specifically, we first retrieve the semantic feature values associated with a specific concept label using the word embedding and we use our visual-semantic mapping to retrieve the associated CNN feature values. Importantly, these values can then be supplied for reconstruction, with the resulting image being an approximate visualization of the mental representation of the concept.

a

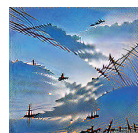

Sky

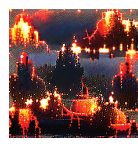

Fire

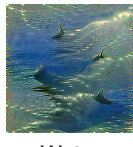

Water

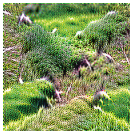

Grass

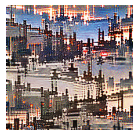

City

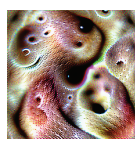

Eye

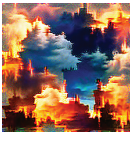

Smoke

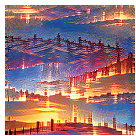

Sunset

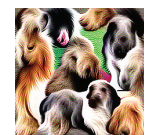

Dog

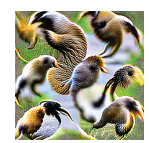

Bird

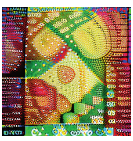

Color

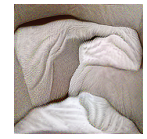

Blanket

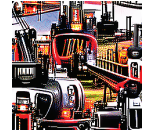

Train

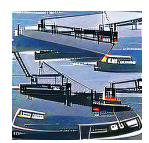

Boat

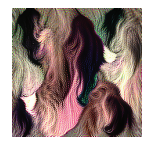

Hair

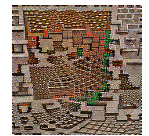

Tile

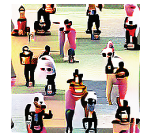

Person

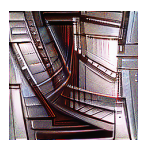

Stairs

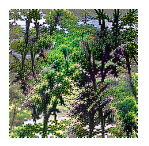

Tree

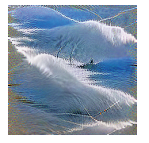

Waves

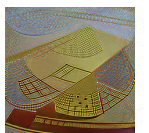

Pyramid

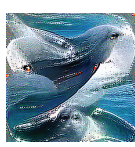

Whale

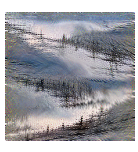

Storm

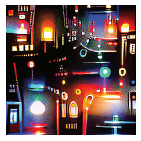

Lights b

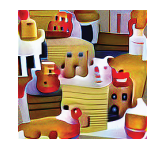

Pizza

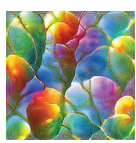

Orange

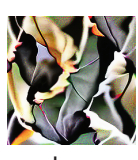

Leg

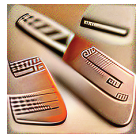

Shoe

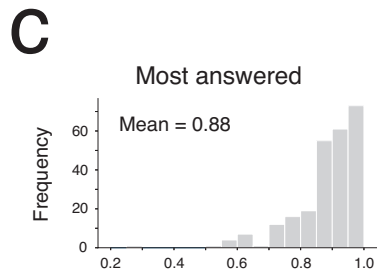

Visual Genome
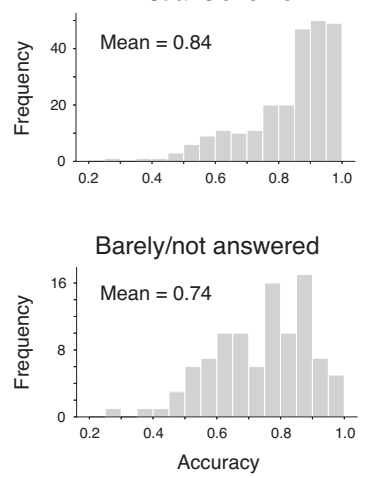

Figure 3. Reconstructions of mental representations and validation. Note that the target concept may be tiled across the image. A) Examples of successful reconstructions (accuracy $>75 \% ; \mathrm{d}_{\mathrm{z}}>1.73 ; \mathrm{p}<.05$, one-sided, FWER-corrected.) B) Examples of failed reconstructions. C) Histograms of recognition accuracies for concepts most named in the main experiment (top), concepts present in the Visual Genome image database (middle), and concepts from the Visual Genome database answered less than 10 times in the main experiment (bottom) (all mean accuracies significant, $\mathrm{p}<.001)$.

We validated the reconstructed representations of 350 words in an additional behavioral experiment: the 250 words most-named in the experiment and the top 100 most frequent words from the Visual Genome database ${ }^{38}$ labeled less than 10 times by participants. On each trial, 50 participants were shown a reconstruction and had to choose between 2 labels: the true label and an incorrect label 
randomly chosen among the labels of the other reconstructions ${ }^{24}$. The reconstructions for the 250 mostnamed words were recognized well on average, with a mean accuracy of $88 \%$ (significantly above chance, $\mathrm{p}<.001)$. The mean accuracy decreased to $84 \%(\mathrm{p}<.001)$ for all labels present in the Visual Genome database, irrespective of whether they were named during the experiment (209 concepts). For the 100 concepts from the Visual Genome database labeled less than 10 total times by participants, the mean accuracy remained high at $74 \%(\mathrm{p}<.001)$. Overall, 270 concepts (out of the 350 that were validated) were individually recognized significantly above chance (accuracy $>75 \% ; d_{z}>1.73 ; p<$ .05 , one-sided, FWER-corrected; Figure 3c), including 47 that were rarely or never named by participants. Successful reconstructions and failure cases can be seen on Figure 3 (panels a and b, respectively). Among best recognized concepts were bird, building, and people, with accuracies of $100 \%$. Worst recognized were jeans, white, and feet (38\%, 30\%, and $25 \%$, respectively).

We also performed an additional, more stringent, validation task in which a new set of 50 participants spontaneously labeled 100 reconstructions. Specifically, each participant was shown the reconstructions of the 100 most-named concepts and had to provide 3 potential labels for each reconstruction. Despite the greater difficulty of this task, several concepts were recognized with high accuracy: the most common written label was the correct label for 37 of these concepts $(\mathrm{p}<.001$; significantly above the 1.1 concepts that would be labeled correctly on average by chance). Results were consistent with the first validation study: all 37 concepts were among those successfully recognized in that study. The most successful concepts included bird, tree, and people (correctly answered by 49,47 , and 46 participants, respectively).

Among the large number of concepts that could be reconstructed, it was still possible to distinguish between closely related and visually similar concepts (Figure 4). Interestingly, singular and plural forms of the same concept could also be differentiated, with more smaller repetitions of the concept across the image when the plural form of a given word was input (Figure 4f). Note that 270 does not seem to be the upper bound of the number of concepts that can be reconstructed: other words can be input that result in seemingly successful reconstructions (e.g., pond, bushes and shark on Figure 4), although we cannot know for sure without validating them too. 
a

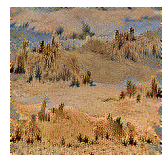

Desert

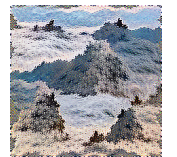

Mountain

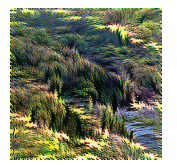

Jungle

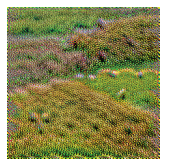

Field

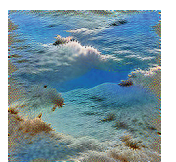

Sea

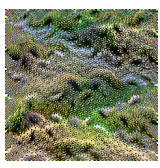

Swamp

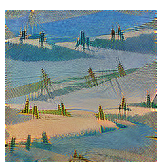

Beach

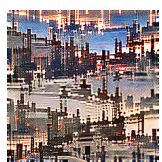

City

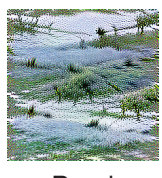

Pond b

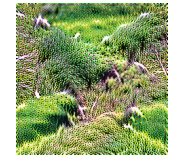

Grass

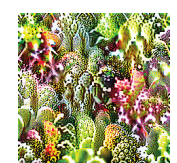

Cactus

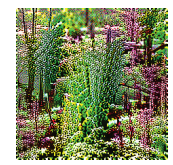

Plant

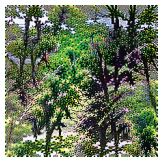

Tree

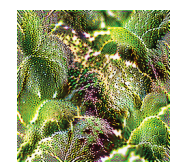

Leaf

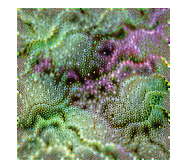

Algae

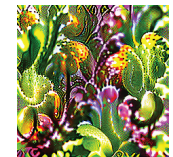

Flower

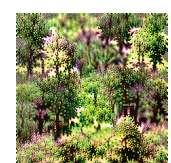

Bushes

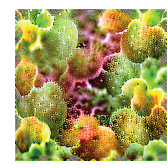

Fruit
C

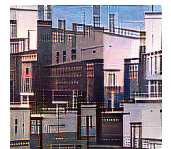

Building

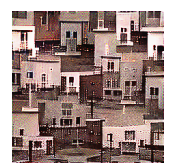

House

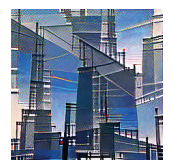

Skyscraper

$f$

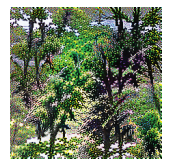

Tree

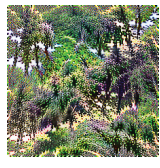

Wheat

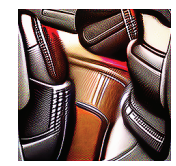

Leather

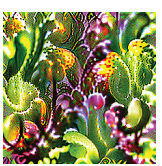

Flower

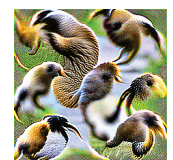

Bird

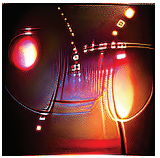

Light

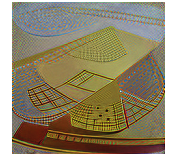

Pyramid

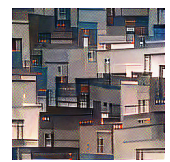

Apartment

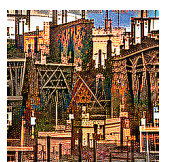

Church

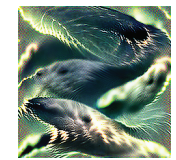

Shark

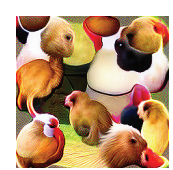

Chicken

e

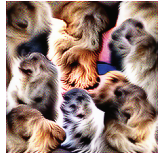

Cat

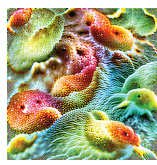

Fish

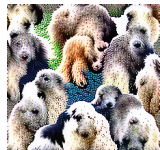

Bear

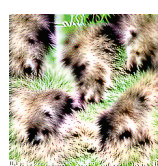

Fox

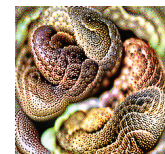

Snake

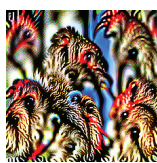

Peacock

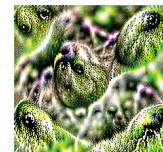

Frog

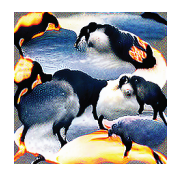

Penguin

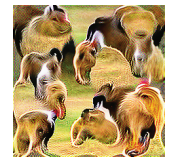

Camel

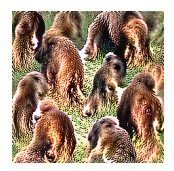

Gorilla

Figure 4. Reconstructions of specific types of concepts. Note that the target concept may be tiled across the image. A) Outdoor scenes. B) Vegetation. C) Buildings. D) Materials and elements. E) Animals. F) Singular/plural concept pairs.

We conducted several control analyses. First, to verify that there was no bias toward the reconstructed concepts inherent in our reconstruction method, we reconstructed concepts after randomly permuting the vectors of semantic features across trials (disrupting visual-semantic associations). As expected, the reconstructions were greatly altered by this shuffling and they varied widely depending on the specific permutation, showing that our results are not an artifact of the method (Figure 5a). Second, to visualize the impact of the random seed used for reconstruction, we 
reconstructed images from the same $\mathrm{CNN}$ feature values using different seeds. Although slight differences can be observed, these concern mostly the location of the features across the image (Figure 5b). Third, we visualized the uncertainty associated with the CNN feature values. To do so, we obtained the lower and upper bounds of $95 \%$ confidence intervals around the $\mathrm{CNN}$ feature values and reconstructed images from these. The true reconstruction is likely to fall between these bounds and so these images give more information about the true representation than the reconstruction from the observed feature values alone (Figure $5 \mathrm{c}$ ).

Fourth, we analyzed the impact of the semantic embedding on reconstructions. We first assessed whether we could reconstruct the representation of a concept without using the concept's name, by relying on other words to learn the visual-semantic mapping. For each reconstruction, we removed all responses that
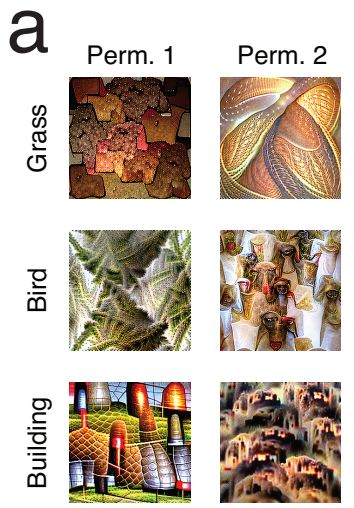

C
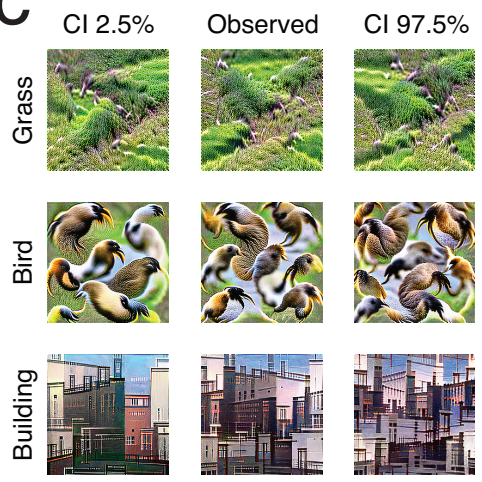

Figure 5. Control reconstructions and analyses, for 3 of the 10 mostnamed concepts. A) Reconstructions using visual-semantic mappings from a permuted null distribution. B) Reconstructions from two different random seeds. C) Reconstructions from the lower and upper bounds of $95 \%$ confidence intervals (C.I.) around the observed CNN feature vectors. D) Reconstructions using visual-semantic mappings created without the reconstructed concept's labels. Correlations between the feature vectors from this analysis and the main analysis for the three depicted concepts are $0.85,0.87$, and 0.92 , respectively. E) Reconstructions using no semantic embedding. Correlations between the feature vectors from this analysis and the main analysis for the three depicted concepts are $0.99,0.97$, and 0.97 , respectively.

contained the concept's name and re-ran all analyses. The resulting reconstructions were quite similar to the originals (Figure 5d), even for the 10 most-named words (correlations of $0.63-0.92$ between the CNN features from both analyses for the top 10 words; $Z=3.29-4.16$; all $\mathrm{p}<.001$, one-sided, FWERcorrected; mean $r=0.85,95 \%$ C.I. $=0.840-0.852$ ). This performance is notable given that we expected these most-named concepts to be most affected by this analysis (because many of the original responses that contributed to their reconstructions were removed), in contrast to less-named concepts for which fewer responses were removed by definition. We then repeated the analyses without using a semantic embedding at all (Figure 5e). Specifically, for each representation to reconstruct, we repeated the analysis while replacing the semantic feature values of a stimulus with a 0 or 1 indicating whether the concept was labeled on that trial (see Methods: Investigation of the effect of the semantic 
embedding). This analysis was feasible only for words that were named frequently. We postulated that, for these frequent words, the representation uncovered by this analysis would closely approximate the true representation. The representations uncovered by our main analysis thus need to be close to these representations. Indeed, reconstructions were highly similar and CNN feature values for the 10 mostnamed concepts were highly correlated with those obtained for these concepts in the main analysis (correlations of 0.91-0.99; $\mathrm{Z}=3.75-5.16$; all $\mathrm{p}<.001$, one-sided, FWER-corrected; mean $\mathrm{r}=0.96$, 95\% C.I. $=0.961-0.964$; Figure 5e). Correlations declined as label frequency decreased (Log frequency explains $64 \%$ of the variance in Fisher-transformed correlation over all words named 10

times or more; $95 \%$ C.I. $=0.633-0.652 ; \mathrm{p}<.001$, one-sided). One advantage of the semantic embedding is to extend beyond these frequent cases to reconstruct representations of less common labels, or even labels not generated in the experiment.

\section{Predicting semantic content and similarity judgments}

We then tested whether our visual-semantic mapping could predict behavior in contexts other than our experiment. Such analyses allow us to infer whether the uncovered mapping is general, i.e., representative of the population and applicable to other situations. We first verified whether it could predict the semantic content of new stimuli. To do so, we computed the semantic feature values associated with the five practice stimuli that all observers saw (and thus for which the semantic content could be estimated reliably) and that were not used to create the mapping. We then tried to predict these feature values based only on the visual content of the images, using our visual-semantic mapping (Figure 6a). We obtained an average cosine similarity of 0.30 (95\% C.I. $=0.17-0.41 ; \mathrm{Z}=20.13 ; \mathrm{p}<$ 
.001 , one-sided; individual cosine similarities ranged from 0.09 to 0.49 ) between the predicted and true semantic feature values, indicating that our mapping can generalize to new stimuli.

We also assessed whether we could predict the stimuli that have been classified by participants as depicting a specific concept. To avoid circularity ${ }^{39}$, we used the set of stimuli and responses from a separate experiment (see 'Revealing representations of single individuals' section). For each concept, we predicted the stimuli most likely to have been classified as containing the concept and compared these predictions to ground truth (Figure 6b). When doing this with the 10 most-named concepts, our predictions were better than chance for 9 of 10 concepts (all except "eyes"; Dice coefficients $=0.19$ $0.64 ; \mathrm{Z}=2.16-11.39 ; \mathrm{p}$ ranged from $<.001$ to .15 , one-sided, FWER-corrected). Even stimuli predicted as containing a concept but for which the concept was not labeled seemed to include it in many cases

a
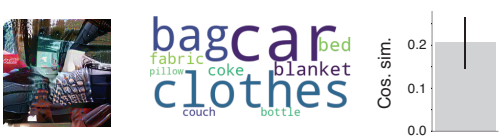

b
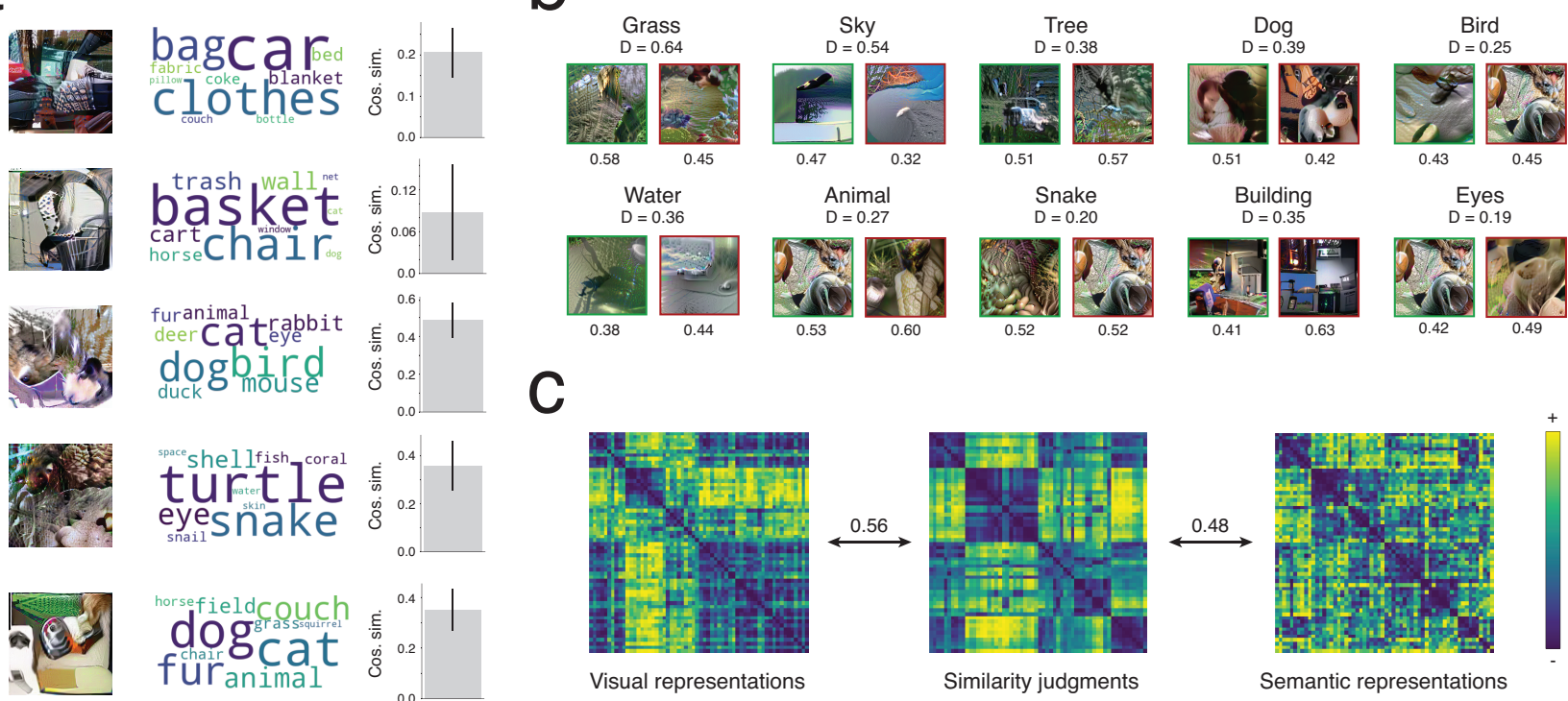

C
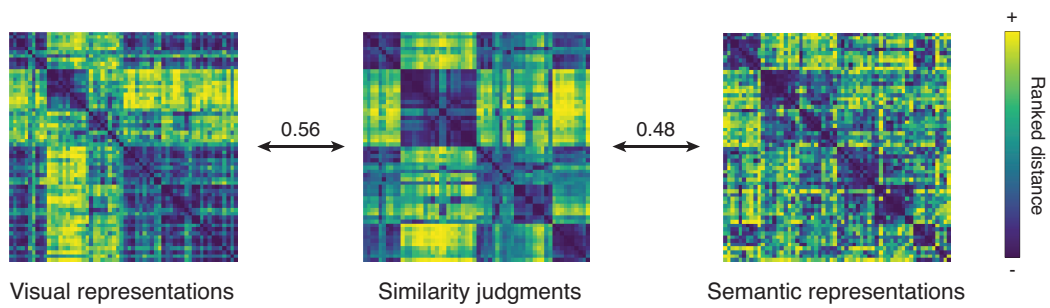

Figure 6. Prediction analyses. A) Prediction of the semantic content of held-out stimuli. Each predicted stimulus is illustrated (left) with associated responses (middle) and the cosine similarity between the true and predicted semantic feature values (right). The average cosine similarity is 0.30 (95\% C.I. $=0.17-0.41 ; \mathrm{Z}=20.13 ; \mathrm{p}<.001$, one-sided) and individual cosine similarities ranged from 0.09 to 0.49. Error bars reflect bootstrap standard errors. B) Prediction of stimuli associated with the 10 most-named concepts. For each concept, stimuli correctly (green frame, left) and incorrectly (red frame, right) predicted as illustrating the concept are depicted. In each case, the depicted stimulus is the one predicted as most likely to include the concept (i.e., whose CNN feature values are most correlated to the CNN feature values of the concept; this correlation is indicated below each stimulus). For each concept, the Dice coefficient, representing the accuracy of the prediction for that concept overall, is below the name of the concept. All Dice coefficients are significant except for "eyes" (0.19-0.64; $Z=2.16-11.39$; p ranged from $<.001$ to .15, one-sided, FWER-corrected). C) Prediction of similarity judgments. The rank-transformed distances between the concepts according to their CNN feature values derived with our visual-semantic mapping (left), to behavioral similarity judgments (middle), and to their semantic feature values derived with the semantic embedding (right). Second-order correlations between the behavioral judgments distance matrix and the other similarity matrices are indicated between the matrices. The visual representations RDM correlated strongly with the similarity judgments RDM $(\rho=0.56 ; 95 \%$ C.I. $=0.458-0.647 ; Z$ $=20.04 ; p<.001$, one-sided $)$ and this correlation was stronger than with the RDM based on semantic representations $(\rho=0.48 ; 95 \%$ C.I. $=0.357-0.591 ;$ significantly weaker, $\mathrm{Z}=2.41 ; \mathrm{p}=.024$, two-sided).

(e.g., see incorrect predictions for grass, sky, tree, and water in Figure 6b). This suggests that some of the prediction errors reflect a misestimation of the semantic content of the stimuli (because of too few participants/responses) rather than an inaccurate visual-semantic mapping. Overall, this result 
indicates that our visual-semantic mapping can be used to infer the semantic content perceived in new images.

Finally, we wanted to explore whether our mapping could predict human behavior in other tasks. To do so, we verified whether the similarity structure of the visual representations uncovered with our visual-semantic mapping predicts how independent observers judge similarity between concepts (Figure 6c). Participants placed written visual concepts on a 2D plane according to their semantic similarity (data from ref. ${ }^{40}$ ). Even though participants were instructed to use semantic information to make their judgments, we predicted that they would still use visual information and that we might capture a unique part of the total variance. Indeed, the visual representations RDM correlated strongly with the similarity judgments $\operatorname{RDM}(\rho=0.56 ; 95 \%$ C.I. $=0.458-0.647 ; Z=20.04 ; \mathrm{p}<.001$, one-sided) and this correlation was stronger than with the RDM based on semantic representations ( $\rho$ $=0.48 ; 95 \%$ C.I. $=0.357-0.591$; significantly weaker, $Z=2.41 ; p=.024$, two-sided), indicating that visual similarities better explain semantic similarity judgments than semantic embeddings trained on word co-occurrences. Together, these results suggest that representations uncovered with our visualsemantic mapping generalize beyond our observers, task and stimuli.

\section{Investigating the representations of the convolutional neural network}

We then aimed to determine whether human representations were different from the representations of the network used for image synthesis. This is important for several reasons. First, uncovering differences between human and CNN representations would show the added value of our method and that using $\mathrm{CNN}$ representations as a proxy for human representations is insufficient. Moreover, it would reveal that our method is a useful tool to analyze representations of CNNs, in addition to human representations, and potentially to compare representations of different CNNs to each other (to identify

the ones that capture behavior better). To achieve this, we repeated the experiment but used as the responses for each stimulus the labels of the three classes (out of the 1000 ImageNet classes) that the network estimated had the highest probability of being depicted in that stimulus. We reconstructed the network's representations in the same way as we did for the human representations. Resulting reconstructions often look superficially similar to reconstructions of human representations but with the concept rendered less clear or even unidentifiable (Figure 7a). We then analyzed whether representations were significantly different between groups (humans vs. CNN) while accounting for different noise levels. Specifically, we projected the visual-semantic mappings of both humans (Figure 
7b) and the network (Figure 7c) to a common semantic space, divided the data in halves, and compared within-group correlations of $\mathrm{CNN}$ feature values to between-group correlations. $\mathrm{CNN}$ feature values were more correlated within group $(\mathrm{r}=0.70$ vs $0.48 ; 95 \%$ C.I. $=0.701-0.705$ vs $0.477-0.483 ; \mathrm{Z}=$ 8.73; $\mathrm{p}<.002$, two-sided), suggesting limits in the correspondence between CNNs and humans (see also ref. $\left.{ }^{41}\right)$.

a
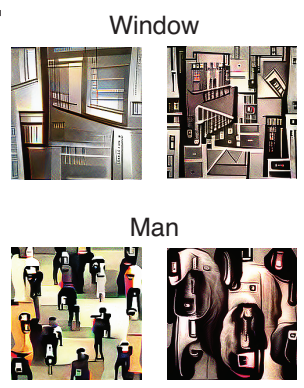

Tree

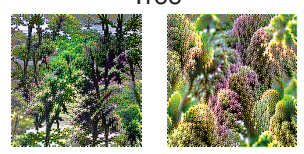

Shirt

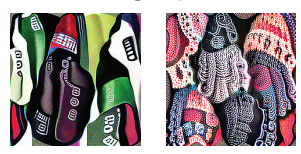

Person

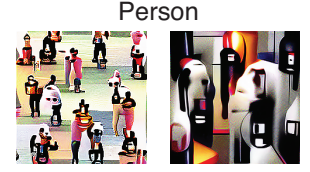

Wall
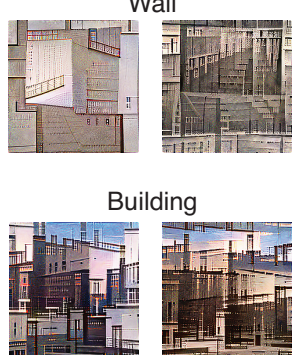

Ground

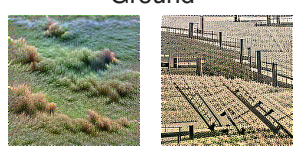

Sky

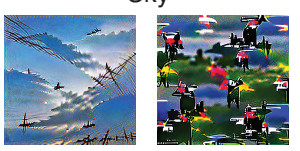

Sign

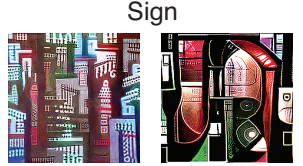

b

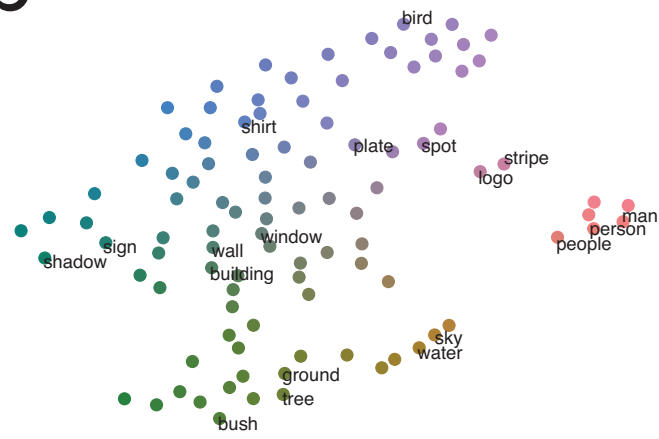

C

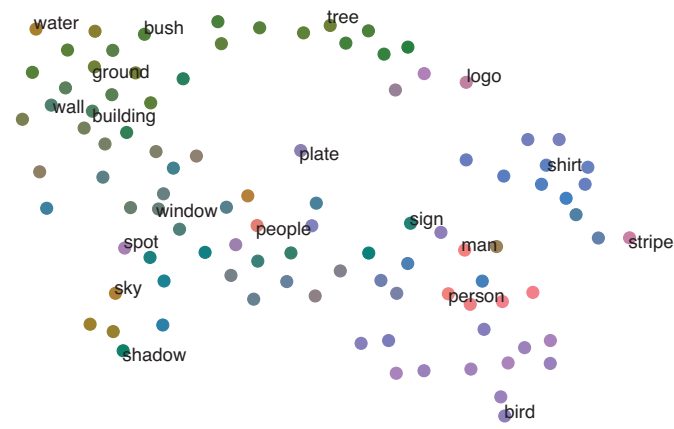

Figure 7. Investigating the representations of the convolutional neural network. A) Reconstructions of human (left) and CNN (right) representations for the top 10 concepts from the Visual Genome database. B) t-SNE plot summarizing the structure of the human representations of the top 100 concepts from the Visual Genome image database. Labels highlight the 10 concepts depicted in panel A and selected additional concepts. Colors gradually change in both axes. C) Same as panel B for the CNN representations. The concepts highlighted with labels and the associations between colors and concepts match panel B, allowing for comparison of relative positions. Overall, $\mathrm{CNN}$ feature values were more correlated within group than between groups (humans vs CNN; $r=0.70$ vs $0.48 ; 95 \%$ C.I. $=0.701-0.705$ vs $0.477-$ $0.483 ; \mathrm{Z}=8.73 ; \mathrm{p}<.002$, two-sided), indicating that representations were different. 


\section{Revealing representations of single individuals}

Interindividual differences in mental representations have been documented in several cases ${ }^{11,42-43}$. Being able to reveal such differences is a strength of methods using human data (behavioral, as in reverse correlation experiments, or neural, as in brain reconstruction paradigms). Using a neural network or other model as a direct way to generate images, despite having tremendous value, will never capture such representational idiosyncrasies. Indeed, a single model cannot
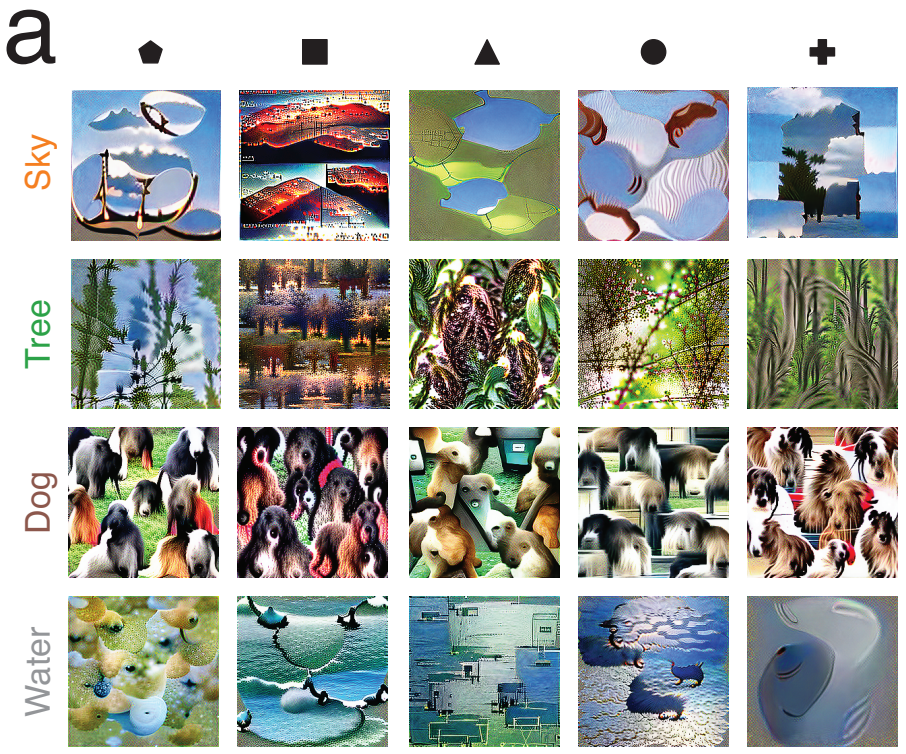
capture the subtle biases and particularities of the representations of specific individuals. To assess whether our method can reveal how conceptual representations vary across individuals, we asked 8 observers to each perform 750 trials of the experiment over the course of several days (all observers saw the same stimuli). We reconstructed representations as before but now for each individual. For the most frequently labeled concepts, reconstructions largely succeeded despite visibly higher levels of noise (Figure

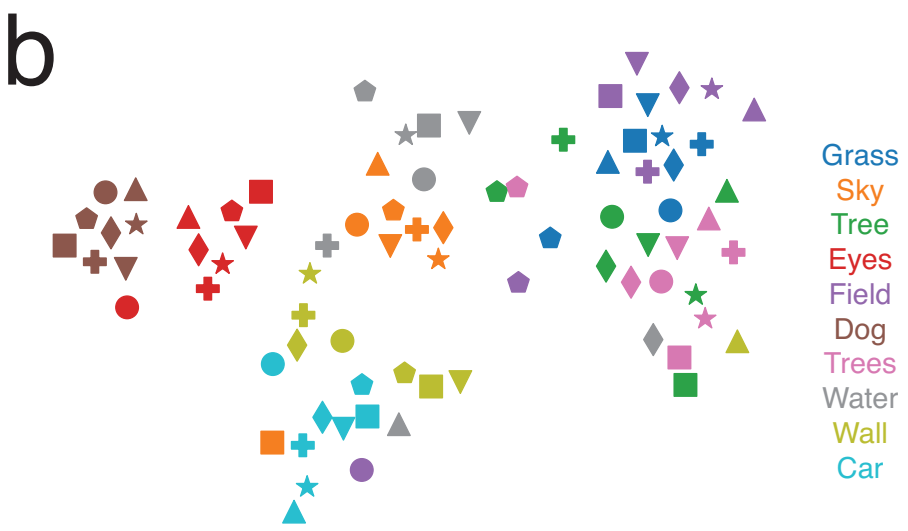

Figure 8. Reconstructing individual representations. A) Reconstructions of selected concepts for five participants. Shape symbols and word colors refer to panel B. B) t-SNE plot summarizing the representational structure of the 10 most-named concepts (colors; see legend on the right) for all 8 participants (shapes). Overall, $\mathrm{CNN}$ feature values were more similar within individual than between individuals $(\mathrm{r}=0.22$ vs $0.11 ; 95 \%$ C.I. $=0.195-0.243$ vs $0.104-0.124 ; Z=15.00, p<.002$, two-sided), indicating that representations were individually unique.

8a). When visualizing an approximation of the structure of all representations on a 2D plane, representations of a given concept for different participants were clustered (Figure 8b). Nevertheless, there were differences, as representations for some concepts were more scattered than others (e.g., water, field, and sky in Figure 8b). To assess whether representations were truly individually unique and stable, we performed an analysis like the one performed to compare human and CNN representations, comparing within-individual to between-individual correlations of $\mathrm{CNN}$ feature values. $\mathrm{CNN}$ feature values were more similar within individual $(\mathrm{r}=0.22$ vs $0.11 ; 95 \%$ C.I. $=0.195-$ 
0.243 vs $0.104-0.124 ; Z=15.00, p<.002$, two-sided), creating the possiblity of using this approach to characterize individual differences in visual concepts and semantic memory. Future research should investigate these representational differences, their origins, and their impact on behavior (e.g., accuracy and response times in object recognition tasks) in more detail.

\section{Discussion}

In this study, we synthesized images that were random mixes of abstract visual features, mapped these features to visual categories, and visualized mental representations of these categories. To our knowledge, our study is the first large-scale attempt at reconstructing the representations of all natural visual concepts. It is also the first time that mental representations of complex natural categories have been reconstructed from purely behavioral responses.

Our method allowed us to successfully reconstruct the representations of at least 270 concepts. We were able to recover the representations of diverse concepts, spanning multiple domains such as animals, vegetation, buildings, colors, materials, and objects. Moreover, we recovered distinct representations of closely related concepts such as grass, plant, and leaf, and were able to differentiate, at least in some cases, between the plural and singular forms of the same base concept. Importantly, 270 is probably not the upper limit for the number of concepts we can reconstruct. We could only validate a finite subset of all possible representations, and it appears that the reconstruction procedure worked with additional unvalidated concepts.

In addition, we were also successful in predicting the semantic content of held-out stimuli and behavioral similarity judgments from separate observers. These results suggest that our visualsemantic mapping generalizes beyond the confines of our experimental data to new stimuli, observers and tasks. Furthermore, we could recover representations of the neural network used in this study and of individual observers. We observed that the representations of the CNN were significantly different from those of human observers. This suggests that simply using the CNN's representations as a direct proxy for human representations is not sufficient. In addition to modelling the human representations more accurately, our method could also be used to quantify the differences in conceptual representations between different neural networks or other models. We also showed that our method can be used to assess representational differences across individuals, something that a single general model cannot do. Future studies should characterize these representational differences more fully and assess their impact on behavior, for example, whether these idiosyncrasies affect accuracy or response 
time in categorization or discrimination tasks. The developmental and sociocultural origins of these idiosyncrasies would also be very interesting to investigate.

Successful reconstructions in the main experiment required an average of 37 trials or 80 responses per concept. This is a major improvement over the 20,000 trials required to retrieve the mental representation of a static letter " $s$ " using pixel noise ${ }^{8}$. Recovering so many representations with so few trials was possible because of several methodological choices. First, allowing participants to give multiple responses on each trial resulted in multiple data points per stimulus. The cost in time for this increased information was smaller than increasing the number of stimuli similarly (especially given automatic suggestions of word completions that may have accelerated response entry for slow typers). Second, using CNN features instead of pixels as features dramatically constrained the search space of our experiment. Not only is this feature space lower-dimensional than the images $(1,024$ channels vs. 50,176 pixels), but it is more proximal to the concepts we sought to reconstruct. The features represent object parts or complex textures that are closer to the natural visual categories than individual pixels. Of course, the ultimate goal is then to recreate a pixel image from these CNN features, but this synthesis process is completely separate from the behavioral experiment itself and it does not rely on any human data. As a corollary, the representations of different concepts are likely more separable in that space ${ }^{44-45}$. Third, treating each response word as part of a continuous multidimensional space allowed us to exploit relationships between words without needing additional trials to retrieve the representation of each word. Indeed, in our analyses, visual features were associated with dimensions of the multidimensional semantic space (i.e., semantic features) rather than specific words. Thus, to reconstruct the representation of one concept, we benefitted not only from trials in which that concept's name was reported, but from all trials. Fourth, we further reduced the dimensionality of both the sampled visual space and the semantic response space by performing principal component analyses.

Our paradigm is based on the idea of reverse correlation ${ }^{5-6}$ in which some features are randomly sampled on every trial. This allows us to uncover the features associated with a target concept in an unbiased way. If we were to show natural images of concepts instead of random features, our reconstructed representations would be biased by this set of natural images. There are internal biases in our representations that are not present in natural images: we typically focus on (and therefore remember) some features more than others (e.g., ref. ${ }^{46}$ ). Using natural images would also prevent us from uncovering interindividual differences in representations, because most natural images would be labelled the same way by all observers. Interindividual differences may be mostly visible in the distinct 
weighting of specific features, and they would likely not be uncovered if the images shown comprised all relevant features. Our CNN-noise stimuli force the participants to rely on subtle features that may be perceived differently across observers and allow us to uncover the "edges" of the representations that better reveal individual variation (in contrast to their centers, likely to be agreed upon by everyone). Finally, such an approach would not allow us to recover representations of concepts with no associated natural images, such as "dragon" or "ghost" or "strange". In sum, using random features allowed us to recover good reconstructions of multiple concepts because we could sample the relevant feature space in an exhaustive and unbiased way.

Note that conceptual representations live in a more abstract space than the image space, with multiple images linked to a given concept. Our reconstructions are thus approximate visualizations i.e., projections onto the image plane - of the mental representations. Specifically, our reconstructed image for a concept visualizes the CNN features most strongly associated with that concept. Indeed, the reconstruction is optimized from the recovered feature values of the category, indicating how much each $\mathrm{CNN}$ feature is associated with the concept. If, for example, leaves are strongly associated to the concept "tree" but pinecones less so, the reconstruction will depict a deciduous tree rather than a conifer, although both are part of the concept "tree" in the minds of the observers.

Despite its success and potential, there are some limits to our method. Notably, whereas sampling pixels involves directly varying the value of the elements that constitute the image, sampling abstract $\mathrm{CNN}$ features requires choices about how to design the features that may not be optimal. Although pixels can allow the recovery of any image, using more complex features reduces the space of possible images and introduces some biases. These apparent drawbacks are balanced by other, more positive consequences. First, the greater expressiveness of pixels is only theoretical: in practice, it would require an unreasonable number of trials to recover representations of complex targets. Second, we are not simply seeking to reconstruct existing 2D images. As noted above, representations of highlevel concepts live in a more abstract feature space, and so we are first aiming to recover representations in that feature space, and only afterwards recreating an image from that representation. This last step could be modified to recreate different - or even multiple - images, without altering the reverse correlation aspect of the paradigm that operates in the abstract feature space. Finally, and most importantly, $\mathrm{CNN}$ features are likely the most appropriate features to sample to retrieve representations of natural concepts. In computer vision, CNNs are typically trained to categorize objects using millions of image-label pairs. In this process, the different layers of the networks learn increasingly abstract features that ultimately map combinations of raw pixels to one of multiple categories. These networks 
perform this task with near-human or superhuman accuracy ${ }^{47-49}$, suggesting that the features they learn are a good representation of natural images. Moreover, CNN features of images achieve state-of-theart accuracy at predicting the brain activity of participants viewing these images, with higher layers better fit to higher-level brain areas ${ }^{17-19,33,50}$. This indicates that these features are a reasonable approximation of the features represented by the brain. Importantly, the features of these networks are not hand-designed but rather learned directly from the data, minimizing potential biases. However, the layer must be chosen with care: it must not be too close to whole objects, to allow for the discovery of minimally biased representations, and not too close to raw pixels, to be more evocative of categories and allow for a more efficient search for representations. In future iterations of this paradigm, other layers could be chosen, or different layers could be combined across trials or even within trial. Layers of other CNNs trained for object categorization (e.g., the more recent FixEfficientNet ${ }^{51}$ or Vision Transformer ${ }^{52-53}$ ) or of deep neural networks trained for other objectives, such as generative adversarial networks (GANs) ${ }^{24,54}$, could also be used as the feature space. Training the networks on datasets that more closely resemble the distribution of natural categories relevant to humans might further improve the results ${ }^{55}$.

Using a word embedding in the analysis also entails caveats. As with the choice of a visual feature space, a semantic space that is not a good fit to how human representations are structured might hinder the recovery of accurate representations for some categories. For example, if the categories "forest" and "tree" are not close in the semantic space but "forest" and "house" happen to be, the representation of the concept "forest" might be biased toward the representation of the concept "house", especially if the word "forest" itself has not been responded much. These word embeddings are trained on billions of words, and they excel at performing various types of natural language processing tasks ${ }^{56-57}$, indicating that they capture real and useful semantic relationships between the words. Moreover, they can capture at least some aspects of semantic representations in the human brain $^{18,40,58-60}$. Most importantly, our analyses suggest that the word embedding modeled the human representational space reasonably well: the representations we uncovered were similar to representations uncovered without using an embedding, and the embedding allowed us to recover representations of concepts even without using the concept's name in the analysis (Figure 5). It is possible however that embeddings trained on different data (e.g., visual co-occurrences, visual similarities or human similarity judgments) ${ }^{34,61-63}$ would model the structure of the representations even better. Although it would likely require more data, a nonlinear regression could also provide a better mapping between visual and semantic features. The method could also be extended to entire 
sentences from individual words by asking participants to write sentences and by using a sentence embedding (e.g., refs. ${ }^{64-65}$ ) in the analyses. This extension might require other improvements to the paradigm, but if successful, would open new possibilities. Notably, novel representations that are compositions of simpler ones (e.g., "hat on a dog" from "hat", "on" and "dog") could be reconstructed, even if these simpler concepts have only been written in response to distinct stimuli.

A key advance of this work is the ability to investigate conceptual representations in their totality (modulo the caveats above), rather than focusing on a few specific concepts. Characterizing whole representational spaces is a central theme of cognitive computational neuroscience ${ }^{66}$. Unlike representational similarity analysis $(\mathrm{RSA})^{67}$, our method not only characterizes the internal structure of these spaces (i.e., the representational geometry), but also the exact positions of the representations within them (i.e., the representational content, or the feature values associated with each concept). Note that such positions cannot be derived from representational distances; there are in fact an infinity of representational spaces resulting in the same representational geometry. This additional information is necessary to reconstruct and predict representations. In that sense, our method is more akin to encoding models used with neural data ${ }^{68-69}$. However, it is unclear whether encoding models fit exclusively on brain activity could retrieve unbiased mental representations of visual categories.

In addition to investigating individual differences in representational content, this approach could be used to investigate representational differences across development, expertise levels, and cultures. It could also provide a platform for answering questions about mental representations more generally, such as differences between observed and optimal representations, the relationship between category and exemplar representations, and the influence of different experiences on represented content. The stimulus synthesis procedure alone could also be used in conjunction with other behavioral tasks or neuroimaging experiments to answer additional questions. The objective function could be modified to edit the features of otherwise natural images in a systematic way or to synthesize artificial images with varying representational similarity ${ }^{70}$. Additional analyses could also be performed on the data collected from this experiment. Notably, even though CNN features were manipulated, we analyzed other visual features of the reconstructions including pixels, color channels, and the Fourier power spectrum (see Figure S1).

In summary, we mapped visual features to semantic features to characterize the representational space of natural categories. This allowed us to reconstruct images to visualize representations of many concepts in that space, even in individual participants. We also showed that conceptual representations in humans differ from those in the neural network used to synthesize the stimuli. Finally, the 
reconstructed conceptual representations generalized to new stimuli and to a new task. This new framework enables, for the first time, a global characterization of representational content.

\section{Methods}

\section{Main experiment}

The following experimental details were preregistered (https://aspredicted.org/QIN_RFK): sample size, exclusion criteria, experimental design, word embedding, CNN and layer, regression analyses, image reconstruction procedure, and analysis of network representations. We deviated from the preregistration in two minor ways: we used a different word embedding (reconstructions with the original embedding are very similar; see Figure S2) and we used an external dataset (Visual Genome) instead of a cross-validation procedure to choose concepts to reconstruct. All code was written in Python, using the NumPy (https://numpy.org) and PyTorch (https://pytorch.org) libraries. Data collection and analysis were not performed blind to the conditions of the experiment.

\section{Participants}

Participants (healthy adults, aged 18-35, with normal or corrected-to-normal vision) were recruited via the Prolific platform ${ }^{71}$ (https://prolific.co) until we got a final sample size of 100 after exclusions. No statistical methods were used to determine the sample size. Participants that did not complete the experiment from beginning to end or that wrote too many non-concrete words (more than $25 \%$ of words with a concreteness rating inferior to 4 , as assessed by ref. ${ }^{72}$ ) were excluded. Participants provided informed consent to a protocol approved by the Yale University Human Subjects Committee and were compensated $5 \$$ for their participation.

\section{CNN-noise stimuli}

For stimulus synthesis, we used an instance of the ResNet-50 convolutional neural network ${ }^{73}$ that was trained for object categorization on the ImageNet Large-Scale Visual Recognition Challenge (ILSVRC) 2012 dataset $^{48}$ while being robust to adversarial examples (L2 adversarial loss, eps = 3.0; available at https://git.io/robust-reps) ${ }^{74}$. Robust training seems to be necessary (along with the optimization procedure described below) to synthesize clear non-adversarial features ${ }^{74-75}$. Specifically, we chose the $37^{\text {th }}$ layer of the network (last layer of the $4^{\text {th }}$ stage) as our feature space. We aimed to 
choose a layer that would represent relatively high-level features (and allow us to synthesize evocative stimuli) while not being too close to whole objects or concepts (which would constrain our search space too much).

The activations of the layer to all images of the ILSVRC 2012 validation set were first collected and averaged within channels (therefore ignoring spatial location). The resulting channel activations were standardized across images and the covariance matrix of the channels was estimated with optimal shrinkage toward the diagonal matrix ${ }^{76}$. A ZCA/Mahalanobis whitening transform was estimated and applied to the standardized activations to decorrelate the features. The distribution of activations to the validation set images was estimated for each feature in this whitened space using Gaussian kernel density. For each stimulus to synthesize, random values were drawn from the estimated distributions for all features; these random feature values were then colored (i.e., the inverse of the whitening transform was applied) and unstandardized to get back to the original space of the layer channels: these are our target $\mathrm{CNN}$ feature values.

We then synthesized a stimulus from these feature values using iterative optimization. We refer to this stimulus as a "CNN-noise" stimulus because it is associated with approximately random values in the space of $\mathrm{CNN}$ features (in contrast to pixel noise which would represent random pixel luminance variations). Specifically, we adapted the activation maximization algorithm from ref. ${ }^{15}$. That is, we first set random complex Fourier coefficients, drawing from a Gaussian distribution with a standard deviation of 0.01 . Then, on each iteration of the optimization procedure, (i) the coefficients were normalized according to their frequency (1/f scaling); (ii) they were inverse Fourier transformed; (iii) the color channels of the resulting image were decorrelated using the Cholesky decomposition of their covariance matrix estimated using the ILSVRC 2012 training set; (iv) the resulting image was fed to the $\mathrm{CNN}$; (v) the mean squared error between the activations of the layer of interest and the target CNN feature values was computed; and (vi) the gradients with respect to the Fourier coefficients were estimated and applied using the Adam optimizer ${ }^{77-78}$ (weight decay $=0.1$; learning rate $=0.05 ; \beta_{1}=$ $\left.0.9 ; \beta_{2}=0.999\right)$. After 1500 iterations, the final Fourier coefficients were inverse Fourier transformed and the color channels of the resulting image were once again decorrelated: this results in the final optimized CNN-noise stimulus. The median reconstruction $\mathrm{R}^{2}$ (comparing the target $\mathrm{CNN}$ feature values with the $\mathrm{CNN}$ feature values of the final images) was 0.93 . The final $\mathrm{CNN}$ feature values rather than the original ones were used in all analyses. All stimuli used in the experiment (excluding the practice trials) were different, even across participants, to sample the feature space exhaustively. 


\section{Experimental design}

The experiment was programmed using the PsychoPy (Python) and PsychoJS (Javascript) libraries (https://www.psychopy.org) ${ }^{79}$, and it was carried out online on the Pavlovia website (https://pavlovia.org). Prior to the task, participants were provided with detailed written instructions. They were told that "dream-like pictures" would be shown to them, that "unclear or distorted objects, object parts and textures may be seen in the pictures" and that they would need to indicate the "objects or concrete things" that they saw, between 1 and 3 for each picture. They were further asked to be as concise as possible and to use only one noun per thing whenever possible. Participants were asked to stay at one arm's length from their screen (the experiment could not be performed on mobile devices), and they were asked to match the length of a segment on the screen to the length of a debit/credit card they owned to calibrate the size of the stimuli to 6 degrees of visual angle. Participants then needed to perform 5 practice trials (identical across participants) before completing 100 experimental trials (different across participants) in blocks of 20. Between blocks, participants were reminded of the instructions; they could start the next block whenever they were ready to do so. At the end of the experiment, a message was displayed, and participants were redirected to the Prolific website.

On each trial, a mid-gray screen was shown for $200 \mathrm{~ms}$, followed by the CNN-noise stimulus centered on a mid-gray background for $5 \mathrm{~s}$, followed by a mid-gray screen. It is at that moment that participants needed to indicate the visual concepts (min. 1) they perceived in the previous stimulus. Participants had no time limit to enter the labels but were told to answer quickly. To speed up the process, words were automatically suggested based on the characters entered and participants could press a key to accept the suggestions. The words that could be suggested initially consisted of the words with a concreteness rating of at least 4 in the database compiled by ref. $^{72}$ and they were suggested based on their frequency as compiled in that database. The suggestions were then adapted to the words written by the participant during the experiment. These automatic and adaptive word suggestions were implemented using the Predictionary JavaScript library (https://github.com/asterics/predictionary). Although participants' responses could have been biased by the automatic suggestions on some occasions, we think that this is unlikely to have had a significant impact on the results for two reasons. First, participants likely perceived the concept they intended to report while the stimulus was being shown, before they could begin responding; to accept a different suggestion would require both that it start with the same letters and that it be a better expression of the perceived concept. Second, autocomplete suggestions were not accepted often in practice, a median of only $9.3 \%$ of the time across participants. 


\section{Visual-semantic mapping}

We first removed all stopwords (using the list from the NLTK Python library ${ }^{80}$; https://www.nltk.org), all one-character words and all numbers from the responses. Then, we used the SymSpell library (https://github.com/wolfgarbe/symspell) to automatically correct (using a maximum edit distance of 2) any word that was not recognized by the word embedding used in the analyses (see below). When there was more than one possible correction, we prioritized suggestions that were visual words (defined as being present in the list of labels of the Visual Genome natural image database ${ }^{38}$; https://visualgenome.org). Words that could not be corrected or that were still unrecognized after correction were removed. All remaining words were then transformed to semantic feature values using a pretrained GloVe word embedding. This embedding maps a vocabulary of 400,000 words to 300dimensional vectors based on their co-occurrence in large text corpora; related words typically have similar vectors and different dimensions encode different semantic aspects ${ }^{57}$. When a label was comprised of more than one word, these were considered as a single "word" in the rare case that they were recognized as such by the word embedding; when not recognized as a single word, the words were split and separately transformed into semantic feature values. For each stimulus, the vectors of semantic feature values of all reported words were then averaged into one vector.

Rather than using only the sampled CNN layer for the analyses, we chose to use both the layer that was sampled and a slightly higher layer (43 ${ }^{\text {rd }}$ layer), following testing on pilot data. We averaged activations within each channel and concatenated channel activations from both layers: these were our new CNN feature values. Principal component analyses (PCA) with whitening were then applied to both the CNN feature values and the semantic feature values across trials. This was done both to decorrelate the features and to reduce the dimensionality of the data. In both cases, the minimum number of components to explain $90 \%$ of the variance was kept (CNN: 213, semantic: 127). A linear mapping was then inferred by performing an outer product of the two trial $\times$ principal components (PC) matrices; this is equivalent to a multivariate multiple linear regression given that variables are random and uncorrelated ${ }^{7-8,81}$. The result was a CNN PC $\times$ semantic PC matrix indicating how much each CNN PC correlates to each semantic PC (henceforth, the mapping matrix; Figure 2b). We repeated these analyses 1000 times while randomly permuting the vectors of semantic feature values across trials (thus disrupting the potential associations between responses and stimuli) to establish a null distribution of mapping matrices. To assess the statistical significance of the visual-semantic associations, we computed the maxima across coefficients of the null mapping matrices and we 
extracted the percentile of the actual value in this distribution of maxima as the $p$ value ${ }^{82}$. Such a statistical test allows us to test the statistical significance of all coefficients while relying on fewer assumptions than parametric tests and correct for multiple comparisons using the optimal maximum statistic method. To visualize semantic PCs, we selected the words, among all words responded in the experiment, that loaded most strongly for that PC (Figure 2c). To visualize CNN PCs, we maximized the values in the direction of the vector of feature values corresponding to the CNN PC (using the caricature objective function, see Methods: Reconstruction of mental representations; Figure 2c). The optimization was carried out according to the procedure described in Olah et al. (2017). Additionally, we selected the words that had the highest cosine similarity between their semantic feature values and the semantic feature values associated with the CNN PC (Figure 2d).

\section{Reconstruction of mental representations}

To visualize a mental representation of a concept, we first computed the $\mathrm{CNN}$ feature values associated with this concept. To do so, we obtained the concept's semantic feature values using the word embedding, transformed the semantic feature values to semantic PC values using the fitted semantic PCA, transformed the semantic PC values to visual PC values using the visual-semantic mapping, and transformed the visual PCs to layer channels using the fitted visual PCA.

We then iteratively optimized an image from these target $\mathrm{CNN}$ feature values using a procedure similar to the one employed to generate stimuli, except for two important things. First, we maximized the caricature objective function ${ }^{83}$ (Eq. 1; Cammarata, Olah \& Satyanarayan, in preparation) instead of minimizing the mean squared error:

$$
\mathbf{p}^{*}=\underset{\mathbf{p}}{\arg \max }\langle\mathbf{y}, \phi(\mathbf{p})\rangle\left(\frac{\langle\mathbf{y}, \phi(\mathbf{p})\rangle}{\|\mathbf{y}\|\|\phi(\mathbf{p})\|}\right)^{\alpha}
$$

where $\mathbf{y}$ is the target vector of feature values, $\mathbf{p}$ are the pixel values of an image, $\phi(\mathbf{p})$ is the feature vector associated with these pixel values, $\mathbf{p}^{*}$ are the pixel values of the reconstructed image and $\alpha$ is a free parameter. To summarize, this objective function consists of the dot product between the target CNN feature values and the CNN feature values of the synthesized image, multiplied by the cosine similarity between these vectors of feature values weighted by some arbitrary exponent $\alpha$. This has the effect of maximizing feature values in the direction of the target $\mathrm{CNN}$ feature values in highdimensional feature space, instead of trying to match it exactly; the cosine similarity term ensures that the direction stays reasonably close to the target direction (the strength of this constraint can be varied by adjusting the $\alpha$ exponent; here, $\alpha$ was set to 4 , following testing on pilot data). We used this 
objective function because the magnitude of the target feature vector is arbitrary, the optimal magnitude for reconstruction is unknown, and maximizing feature values allows us to visualize the important features of the representation most clearly. Images were optimized for 2000 iterations using a learning rate of 0.05 . A second important difference is that we use transformation robustness in this reconstruction procedure; that is, at each iteration of the optimization process, the image is subjected to small rotations, translations and homothecies prior to be fed to the network. This has been found to reduce high-frequency artifacts and improve the clarity of the optimized images ${ }^{15}$.

To visualize the uncertainty intrinsic to the representations, we synthesized images from the CNN feature values that correspond to the lower and upper bounds of $95 \%$ confidence intervals around the CNN feature values associated with some concepts (Figure 5c). To do so, we inferred new visualsemantic mappings while randomly permuting trials (in the same way for both semantic and CNN feature values) with replacement. We then computed the CNN feature values associated with a given concept using each mapping. For each $\mathrm{CNN}$ feature, the lower and upper bounds of the confidence interval corresponded to the $2.5^{\text {th }}$ and $97.5^{\text {th }}$ percentiles of this bootstrap distribution. Images were then synthesized from these $\mathrm{CNN}$ feature values.

\section{Investigation of the effect of the semantic embedding}

To assess the effect of responses containing the concept's name, we repeated for each target concept the creation of a visual-semantic mapping as in the main analysis but ignoring responses with the concept's name. We then computed the concept's CNN feature values using this mapping and reconstructed representations from these using the same procedure as was used in the main analysis (Figure 5d). Finally, we correlated the feature values with the CNN feature values associated with the concept according to the main analysis (Figure S3a). To assess the statistical significance of the correlations, we computed them again but using the null visual-semantic mappings (obtained while randomly permuting the trials 1000 times). The null correlations obtained using this randomization procedure were then used to z-score both observed and null correlations. Finally, we computed the maxima across concepts of the null values and we extracted the percentile of the actual value in this distribution of maxima as the $\mathrm{p}$ value $\mathrm{e}^{82}$. We also computed the bootstrapped estimates of the standard errors of the correlations by repeating the computation of the correlations 1000 times with the dimensions of both variables randomized with replacement.

To assess the impact of the semantic embedding, we repeated the main analysis but replaced the semantic embedding with a binary embedding representing whether each word (among the 369 
words named at least 10 times) was answered or not on a given trial. This allowed us to directly obtain the CNN feature values associated with each of these words. We then correlated these feature values with the CNN feature values of the concepts according to the main analysis (Figure S3b) and we reconstructed the representations of the 10 most-named concepts using the same procedure as was used in the main analysis (Figure 5e). To assess the statistical significance and compute the standard errors, we used similar procedures as for the previous analysis.

\section{Prediction of semantic content}

To predict the semantic content of new images, we obtained the CNN feature values associated with these images by feeding them to the $\mathrm{CNN}$, transformed these into visual PC values using the fitted visual PCA, transformed the visual PC values to semantic PC values using the visual-semantic mapping, and transformed the semantic PC values to semantic feature values using the fitted semantic PCA. We then compared these semantic feature values to the actual semantic feature values from the responses associated with the image using cosine similarity (Figure S4a). To assess statistical significance of the mean cosine similarity, we repeated the above procedure 1000 times while using as responses labels randomly drawn (with replacement) from all labels associated to the tested images, and we extracted the percentile of the actual value in this null distribution as the p value. We also computed the bootstrapped estimates of the standard errors by repeating the computation of the cosine similarities 1000 times with the dimensions of both vectors randomized with replacement.

\section{Prediction of stimuli}

To predict the stimuli associated with a specific concept, we used the visual-semantic mapping from the main experiment and the set of stimuli and responses from the Individual Representations experiment. For each of the 10 most-named concepts (grass, sky, tree, dog, bird, water, animal, snake, building, eyes), we created a Boolean vector indicating which stimuli were associated with the concept at least once. Then, we computed how much the $\mathrm{CNN}$ feature values of each stimulus correlated to the $\mathrm{CNN}$ feature values associated with the concept (as obtained with our visual-semantic mapping). We binarized this vector of correlation coefficients so that the number of stimuli predicted as containing the concept matched the number of stimuli containing it. We then quantified the degree of overlap between the two Boolean vectors by computing their Dice coefficient (Figure S4b). To assess statistical significance, we computed null Dice coefficients by repeating this analysis 1000 times while randomly permuting the order of the stimuli in one variable. We then z-scored the observed and null Dice 
coefficients using the mean and standard deviation of the null Dice coefficients, computed the maxima across concepts of the z-scored null Dice coefficients, and extracted the percentile of the actual value in this distribution of maxima as the $\mathrm{p}$ value ${ }^{82}$.

\section{Prediction of behavioral similarity judgments}

We used the openly available dataset (https://osf.io/um3qg/) used in ref. ${ }^{40}$. In that study, participants are asked to place words describing visual concepts on a $2 \mathrm{D}$ plane according to their semantic similarity (total of 60 words). Subsets of words are shown on each trial in an adaptive fashion, and a representational dissimilarity matrix $(\mathrm{RDM})$ is derived from these judgments using the inverse multidimensional scaling (MDS) algorithm ${ }^{84}$. We rank-transformed RDMs from all participants and averaged them: this is the behavioral judgments RDM. To derive the visual RDM, we obtained the $\mathrm{CNN}$ feature values associated with each of the 60 concepts using the word embedding and our visualsemantic mapping (see Methods: Reconstruction of mental representations) and we computed the correlation distances ( 1 - Pearson correlation) between all vectors of feature values. We used a similar

procedure to derive the semantic RDM, using the semantic feature values associated to each concept instead of the CNN feature values. To assess how well the visual RDM explained the behavioral RDM (and how well the semantic RDM explained the behavioral RDM), we computed the Spearman correlation between the vectorized upper triangles of the matrices. We used Spearman correlation because only the ranks of the distances can be meaningfully compared between matrices ${ }^{67,85}$. We performed a randomization test to assess statistical significance: the Spearman correlation was computed 1,000 times while randomly shuffling rows and columns of the behavioral $\mathrm{RDM}^{67}$ and we extracted the percentile of the actual value in this null distribution as the $\mathrm{p}$ value. We used a nonparametric test because it relies on fewer assumptions than parametric tests and allowed us to keep a coherent statistical approach across analyses. To assess whether the visual RDM correlates more to the behavioral judgments RDM than the semantic RDM, the Spearman correlation was computed for the semantic RDM and the difference between both correlations was computed; a randomization procedure similar to the one described above was applied with the correlation difference to test its statistical significance.

\section{Investigation of the representations of the convolutional neural network}

We had the deep neural network perform the same experiment with the same stimuli as the participants in the main experiment. The labels associated with the top 3 ImageNet classes predicted by the network 
(i.e., the 3 classes with the highest probability) were taken as its responses. When a label comprised multiple synonyms, we used the first one, which was usually the simplest and most common. When a response was not recognized by the word embedding, we went up one level in the WordNet hierarchy to which ImageNet classes are mapped (e.g., from "otterhound" to "hound") and tried again. We used this procedure because ImageNet classes (and therefore the CNN's responses) are sometimes too specific for the word embedding's vocabulary. We analyzed the data in the same way as with the participant data (number of semantic PCs $=118$ ) and we reconstructed the representations of the 10 concepts most common in the Visual Genome database. To visualize the representational spaces, the $\mathrm{CNN}$ feature values associated with the 100 concepts most common in the Visual Genome database were projected on a two-dimensional plane using t-SNE ${ }^{86}$ with correlation distance as metric $($ perplexity $=15)$.

To investigate differences between the "groups" (CNN and humans), we divided the data in halves and computed the visual-semantic mappings for each half and group. We then projected the mappings to an independent semantic space: to do so, we computed for each half and group the CNN feature values associated to each one of the 100 most common concepts from the Visual Genome database (each concept can be viewed as a dimension of a new semantic space). The set of these coefficients summarizes, once flattened, the visual representations of either CNN or humans. We computed the Pearson correlation between the human visual representations in half 1 and the CNN visual representations in half 2 , and the correlation between the human representations in half 2 and the $\mathrm{CNN}$ representations in half 1 . We then averaged these two correlation coefficients to obtain a "between-groups" correlation value. We also computed a within-groups correlation: this is the average of the correlation between the human representations in half 1 and the human representations in half 2 , and the correlation between the $\mathrm{CNN}$ representations in half 1 and the $\mathrm{CNN}$ representations in half

2. The difference between these two average correlations reflects the degree to which the two sets of representations (CNN and human) are unique (e.g., see ref. ${ }^{87}$ ). To assess statistical significance, these analyses were repeated but using the null visual-semantic mappings (obtained while randomly permuting the trials 1000 times) and we extracted the percentile of the actual value in this null distribution as the $\mathrm{p}$ value.

\section{First validation experiment}

The validation experiment was preregistered at https://aspredicted.org/SLR_KFB. 


\section{Participants}

Fifty new participants (healthy adults, aged 18-35, with normal or corrected-to-normal vision) were recruited via the Prolific platform. Participants provided informed consent to a protocol approved by the Yale University Human Subjects Committee and were compensated 3.25\$ for their participation. Two participants were excluded because of mean accuracies more than 3 standard deviations away from the group mean. No statistical methods were used to determine the sample size.

\section{Stimuli}

Reconstructions validated include the reconstructions for the 250 concepts that were most named during the main experiment along with the 100 most frequent concepts in the Visual Genome database $^{38}$ that were named less than 10 times in the main experiment. Reconstructions were obtained using the procedure described above (see Methods: Reconstruction of mental representations).

\section{Experimental design}

The experiment was carried out online on the Pavlovia website (https://pavlovia.org). We used a similar validation procedure as was used in ref. ${ }^{24}$. Prior to the task, participants were provided with detailed instructions: they were told that each depicted concept may be unclear, may occupy the whole image or only a part of it, and may be depicted many times across the image. Participants were also told to keep in mind the different potential meanings of each label. On each trial, a mid-gray screen was shown for $200 \mathrm{~ms}$, followed by a randomly selected reconstruction centered on a mid-gray background. After $1 \mathrm{~s}$, two labels appeared at the top of the screen: the true label and one wrong label chosen randomly among the labels of all other concepts validated in the experiment (different across participants). The two labels were randomly assigned to either the top left corner or the top right corner. Participant needed to indicate at that moment which label most likely corresponded to the image; they could answer with two keyboard keys. There was no time limit, but participants were told to answer quickly. The 350 reconstructions were shown in 7 blocks of 50; participants could take short breaks

between blocks. The presentation order of the stimuli was randomized across participants. Data collection was not performed blind to the conditions of the experiment.

\section{Data analysis}


We computed the mean accuracies across participants for each concept. To assess their statistical significance, we computed them again while randomly permuting the participants with replacement 50,000 times and assessed whether at least 99.993\% (one-tailed 95\% significance threshold with Šidák correction for multiple comparisons across the 350 concepts) of that distribution was above the chance level of $50 \%$. For concepts for which the estimated accuracy separating the rightmost $99.993 \%$ of the distribution from the rest was between $40 \%$ and $60 \%$, the procedure was repeated with 500,000 iterations to improve the precision of the estimation. This statistical procedure estimates the effect for the population of participants while correcting for multiple comparisons across concepts ${ }^{88}$. To assess the statistical significance of the mean accuracies for all concepts or subsets of concepts, we shuffled the trials randomly 1,000 times to establish null distributions. We quantified the $\mathrm{p}$ value as the percentile of the observed mean accuracy in the null distribution. We also assessed the statistical significance using a variant of the above bootstrap procedure (but averaging across multiple objects and using a 95\% threshold). Both statistical methods gave similar results (all mean accuracies significant with $\mathrm{p}<.001)$. Data analysis was not performed blind to the conditions of the experiment.

\section{Second validation experiment}

\section{Participants}

Fifty new participants (healthy adults, aged 18-35, with normal or corrected-to-normal vision) were recruited via the Prolific platform. Participants provided informed consent to a protocol approved by the Yale University Human Subjects Committee and were compensated 7.34\$ for their participation. No statistical methods were used to determine the sample size.

\section{Stimuli}

Reconstructions validated were the reconstructions for the 100 concepts that were most named during the main experiment. Reconstructions were obtained using the procedure described above (see Methods: Reconstruction of mental representations).

\section{Experimental design}

The experiment was carried out online on the Pavlovia website (https://pavlovia.org). Prior to the task, participants were given detailed instructions: They were told that each depicted concept may be unclear, may occupy the whole image or only a part of it, and may be depicted many times across the 
image. Participants were also told that each image is associated to a different label, and that synonyms and different spellings all count as different words. On each trial, a mid-gray screen was shown for $200 \mathrm{~ms}$, followed by a randomly selected reconstruction centered on a mid-gray background for $2 \mathrm{~s}$, followed by another blank screen on which participants could write three guesses about the label of the reconstruction. There was no time limit, but participants were told to answer as fast as possible. When they were done entering their third guess, the next reconstruction was shown. The 100 reconstructions were shown in 5 blocks of 20; participants could take short breaks between blocks. The presentation order of the stimuli was randomized across participants. Data collection was not performed blind to the conditions of the experiment.

\section{Data analysis}

We first corrected spelling mistakes in the participants' responses using the same procedure that was used for the main experiment. For each reconstruction, we then verified whether the correct label was the one most commonly provided by participants. We considered plural and singular forms of a word equivalently. To assess whether the number of successful concepts obtained was significant, we repeated the above analysis 1000 times after randomly permuting participant responses across trials (reconstructions). This resulted in a null distribution of 1000 numbers of "correct" concepts. We extracted the percentile of the actual value in this null distribution as the $p$ value. Data analysis was not performed blind to the conditions of the experiment.

\section{Individual representations experiment}

\section{Participants}

We recruited eight new participants (healthy adults, aged 18-35, 5 women and 3 men, with normal or corrected-to-normal vision) from the Yale University community. Participants provided informed consent to a protocol approved by the Yale University Human Subjects Committee and they obtained a $45 \$$ Amazon gift card for their participation. No statistical methods were used to determine the sample size. No participant was excluded prior to analyses.

\section{Stimuli}


Stimuli were created using the same procedure as in the main experiment. We created 750 stimuli (in addition to 5 stimuli for practice trials) that were used for all participants. Stimuli were identical across participants to facilitate comparisons between them.

\section{Experimental design}

The experiment was carried out online on the Pavlovia website (https://pavlovia.org). The experimental design was the same as in the main experiment except that each participant performed 6 sessions of the experiment, that there were 125 stimuli per session (shown in 5 blocks of 25), and that participants were not redirected to any website at the end of the experiment. The presentation order of the stimuli was randomized across participants. Data collection was not performed blind to the conditions of the experiment.

\section{Data analysis}

For each participant, we created a visual-semantic mapping as described above (see Main experiment: Visual-semantic mapping; number of CNN PCs = 150; number of semantic PCs varied between 55 and 120). We reconstructed individual visual representations using these mappings (Figure 7; see Main experiment: Reconstruction of mental representations). To visualize similarities and dissimilarities between these representations, they were projected on a two-dimensional plane using t-SNE ${ }^{86}$ with correlation distance as metric (perplexity $=15)$.

To assess inter-individual differences in the representations, we used a similar procedure as for the investigation of the network's representations, but we projected the visual-semantic mappings to the independent semantic space defined in the main experiment, and we computed the uniqueness coefficients for each pair of participants and averaged them ${ }^{87}$. Data analysis was not performed blind to the conditions of the experiment. 


\section{References}

1. Marr, D. Vision: A Computational Investigation into the Human Representation and Processing of Visual Information. (Henry Holt and Co., 1982).

2. Pylyshyn, Z. W. Computation and cognition: Issues in the foundations of cognitive science. Behavioral and Brain Sciences 3, 111-132 (1980).

3. Schyns, P. G., Gosselin, F., \& Smith, M. L. Information processing algorithms in the brain. Trends in Cognitive Sciences 13, 20-26 (2009).

4. Wiener, N. Nonlinear Problems in Random Theory. (Wiley, 1958).

5. Ahumada Jr, A. J. Perceptual classification images from Vernier acuity masked by noise. Perception 25, (ECVP Abstract Supplement, 1996).

6. Ahumada Jr, A., \& Lovell, J. Stimulus features in signal detection. Journal of the Acoustical Society of America 49, 1751-1756 (1971).

7. Murray, R. F. Classification images: A review. Journal of Vision 11, 2 (2011).

8. Gosselin, F., \& Schyns, P. G. Superstitious perceptions reveal properties of internal representations. Psychological Science 14, 505-509 (2003).

9. Gosselin, F., Bacon, B. A., \& Mamassian, P. Internal surface representations approximated by reverse correlation. Vision Research 44, 2515-2520 (2004).

10. Morin-Duchesne, X., Gosselin, F., Fiset, D., \& Dupuis-Roy, N. Paper features: A neglected source of information for letter recognition. Journal of Vision 14, 11 (2014).

11. Jack, R. E., Caldara, R., \& Schyns, P. G. Internal representations reveal cultural diversity in expectations of facial expressions of emotion. Journal of Experimental Psychology: General 141, 19-25 (2012).

12. Dotsch, R., \& Todorov, A. Reverse correlating social face perception. Social Psychological and Personality Science 3, 562-571 (2012).

13. Éthier-Majcher, C., Joubert, S., \& Gosselin, F. Reverse correlating trustworthy faces in young and older adults. Frontiers in Psychology 4, 592 (2013).

14. LeCun, Y., Bengio, Y., \& Hinton, G. Deep learning. Nature 521, 436-444 (2015).

15. Olah, C., Mordvintsev, A., \& Schubert, L. Feature visualization. Distill 2, e7 (2017).

16. Zeiler, M. D., \& Fergus, R. Visualizing and understanding convolutional networks. European Conference on Computer Vision, 818-833 (2014). 
17. Cichy, R. M., Khosla, A., Pantazis, D., Torralba, A., \& Oliva, A. Comparison of deep neural networks to spatio-temporal cortical dynamics of human visual object recognition reveals hierarchical correspondence. Scientific Reports 6, 27755 (2016).

18. Güçlu, U., \& van Gerven, M. A. J. Deep neural networks reveal a gradient in the complexity of neural representations across the ventral stream. Journal of Neuroscience 35, 1000510014 (2015).

19. Yamins, D. L. K., et al. Performance-optimized hierarchical models predict neural responses in higher visual cortex. Proceedings of the National Academy of Sciences 111, 8619-8624 (2014).

20. Beliy, R., et al. From voxels to pixels and back: Self-supervision in natural-image reconstruction from fMRI. arXiv:1907.02431 (2019).

21. Gaziv, G., et al. Self-supervised natural image reconstruction and rich semantic classification from brain activity. bioRxiv:2020.09.06.284794 (2020).

22. Ren, Z., et al. Reconstructing seen image from brain activity by visually-guided cognitive representation and adversarial learning. NeuroImage 228, 117602 (2021).

23. Shen, G., Dwivedi, K., Majima, K., Horikawa, T., \& Kamitani, Y. End-to-end deep image reconstruction from human brain activity. Frontiers in Computational Neuroscience 13, 21 (2019a).

24. Shen, G., Horikawa, T., Majima, K., \& Kamitani, Y. Deep image reconstruction from human brain activity. PLoS Computational Biology 15, e1006633-23 (2019b).

25. Bashivan, P., Kar, K., \& DiCarlo, J. J. Neural population control via deep image synthesis. Science 364, eaav9436 (2019).

26. Zijin, G., et al. NeuroGen: Activation optimized image synthesis for discovery neuroscience. NeuroImage 247, 118812 (2022).

27. Senden, M., Emmerling, T. C., van Hoof, R., Frost, M. A., \& Goebel, R. Reconstructing imagined letters from early visual cortex reveals tight topographic correspondence between visual mental imagery and perception. Brain Structure and Function 224, 1167-1183 (2019).

28. Dharmaretnam, D., Foster, C., \& Fyshe, A. Words as a window: Using word embeddings to explore the learned representations of Convolutional Neural Networks. Neural Networks 137, 63-74 (2021). 
29. Frome, A., et al. DeViSE: A Deep Visual-Semantic Embedding Model. Advances in Neural Information Processing Systems 26 (2013).

30. Bengio, Y., Ducharme, R., \& Vincent, P. A neural probabilistic language model. Advances in Neural Information Processing Systems 13 (2000).

31. Huth, A. G., Nishimoto, S., Vu, A. T., \& Gallant, J. L. A continuous semantic space describes the representation of thousands of object and action categories across the human brain. Neuron 76, 1210-1224 (2012).

32. Huth, A. G., de Heer, W. A., Griffiths, T. L., Theunissen, F. E., \& Gallant, J. L. Natural speech reveals the semantic maps that tile human cerebral cortex. Nature 532, 453-458 (2016).

33. Bao, P., She, L., McGill, M., \& Tsao, D. Y. A map of object space in primate inferotemporal cortex. Nature 583, 103-108 (2020).

34. Hebart, M. N., Zheng, C. Y., Pereira, F., \& Baker, C. I. Revealing the multidimensional mental representations of natural objects underlying human similarity judgements. Nature Human Behaviour 4, 1173-1185 (2020).

35. Jha, A., Peterson, J., \& Griffiths, T. L. Extracting low-dimensional psychological representations from convolutional neural networks. arXiv:2005.14363 (2020).

36. Lehky, S. R., Kiani, R., Esteky, H., \& Tanaka, K. Dimensionality of object representations in monkey inferotemporal cortex. Neural Computation 26, 2135-2162 (2014).

37. Olah, C., et al. The building blocks of interpretability. Distill 3, e10 (2018).

38. Krishna, R., et al. Visual genome: Connecting language and vision using crowdsourced dense image annotations. arXiv:1602.07332 (2016).

39. Kriegeskorte, N., Simmons, W. K., Bellgowan, P. S. F., \& Baker, C. I. Circular analysis in systems neuroscience: the dangers of double dipping. Nature Neuroscience 12, 535-540 (2009).

40. Nishida, S., Blanc, A., Maeda, N., Kado, M., \& Nishimoto, S. Behavioral correlates of cortical semantic representations modeled by word vectors. PLOS Computational Biology 17, e1009138 (2021).

41. Xu, Y., \& Vaziri-Pashkam, M. Limits to visual representational correspondence between convolutional neural networks and the human brain. Nature Communications 12, 2065 (2021). 
42. Caplette, L., Wicker, B., \& Gosselin, F. Atypical time course of object recognition in autism spectrum disorder. Scientific Reports 6, 35494 (2016).

43. Tardif, J., et al. Use of face information varies systematically from developmental prosopagnosics to super-recognizers. Psychological Science 30, 300-308 (2019).

44. DiCarlo, J. J., \& Cox, D. D. Untangling invariant object recognition. Trends in Cognitive Sciences 11: 333-341 (2007).

45. Yamins, D. L. K., \& DiCarlo, J. J. Using goal-driven deep learning models to understand sensory cortex. Nature Neuroscience 19, 356-365 (2016).

46. Zhan, J., Garrod, O. G. B., van Rijsbergen, N., \& Schyns, P. G. Modelling face memory reveals task-generalizable representations. Nature Human Behaviour 3, 817-826 (2019).

47. Kheradpisheh, S. R., Ghodrati., M., Ganjtabesh, M., \& Masquelier, T. Deep networks can resemble human feed-forward vision in invariant object recognition. Scientific Reports $\mathbf{6}$, 32672 (2016).

48. Russakovsky, O., et al. ImageNet large scale visual recognition challenge. International Journal of Computer Vision 115, 211-252 (2015).

49. Ho-Phuoc, T. CIFAR10 to compare visual recognition performance between deep neural networks and humans. arXiv:1811.07270 (2018).

50. Storrs, K. R., Kietzmann, T. C., Walther, A., Mehrer, J., \& Kriegeskorte, N. Diverse deep neural networks all predict human inferior cortex well, after training and fitting. Journal of Cognitive Neuroscience 33, 2044-2064 (2020).

51. Touvron, H., Vedaldi, A., Douze, M., \& Jégou, H. Fixing the train-test resolution discrepancy. Advances in Neural information processing systems 32 (2019).

52. Zhai, X., Kolesnikov, A., Houlsby, N., \& Beyer, L. Scaling vision transformers. Proceedings of the IEEE/CVF Conference on Computer Vision and Pattern Recognition, 12104-12113 (2022).

53. Dosovitskiy, A., et al. An image is worth 16x16 words: Transformers for image recognition at scale. arXiv:2010.11929 (2020).

54. Goodfellow, I., et al. Generative adversarial networks. Communications of the ACM 63, 139-144 (2020). 
55. Mehrer, J., Spoerer, C. J., Jones, E. C., Kriegeskorte, N., \& Kietzmann, T. C. An ecologically motivated image dataset for deep learning yields better models of human vision. Proceedings of the National Academy of Sciences 118, e2011417118 (2021).

56. Mikolov, T., Chen, K., Corrado, G., \& Dean, J. Efficient estimation of word representations in vector space. arXiv:1301.3781 (2013).

57. Pennington, J., Socher, R., \& Manning, C. D. Glove: Global vectors for word representation. Proceedings of the 2014 Conference on Empirical Methods in Natural Language Processing, 1532-1543 (2014).

58. Nishida, S., \& Nishimoto, S. Decoding naturalistic experiences from human brain activity via distributed representations of words. NeuroImage 180, 232-242 (2018).

59. Pereira, F., Lou, B., Pritchett, B., Ritter, S., Gershman, S. J., Kanwisher, N., et al. Toward a universal decoder of linguistic meaning from brain activation. Nature Communications $\mathbf{9}$, 963 (2018).

60. Wang, S., Zhang, J., Wang, H., Lin, N., \& Zong, C. Fine-grained neural decoding with distributed word representations. Information Sciences 507, 256-272 (2020).

61. Gupta, T., Schwing, A., \& Hoiem, D. Vico: Word embeddings from visual co-occurrences. Proceedings of the IEEE/CVF International Conference on Computer Vision, 7425-7434 (2019).

62. Hasegawa, M., Kobayashi, T., \& Hayashi, Y. Incorporating visual features into word embeddings: A bimodal autoencoder-based approach. International Conference on Computational Semantics (2017).

63. Roads, B. D., \& Love, B. C. Enriching ImageNet with human similarity judgments and psychological embeddings. Proceedings of the IEEE/CVF Conference on Computer Vision and Pattern Recognition, 3547-3557 (2021).

64. Devlin, J., Chang, M., Lee, K., \& Toutanova, K. BERT: Pre-training of Deep Bidirectional Transformers for Language Understanding. arXiv:1810.04805v2 (2018).

65. Reimers, N., \& Gurevych, I. Sentence-bert: Sentence embeddings using Siamese bertnetworks. arXiv:1908.10084 (2019).

66. Kriegeskorte, N., \& Douglas, P. K. Cognitive computational neuroscience. Nature Neuroscience 21, 1148-1160 (2018). 
67. Kriegeskorte, N., Mur, M., \& Bandettini, P. Representational similarity analysis connecting the branches of systems neuroscience. Frontiers in Systems Neuroscience 2, 4 (2008).

68. Naselaris, T., Kay, K. N., Nishimoto, S., \& Gallant, J. L. Encoding and decoding in fMRI. NeuroImage 56, 400-410 (2011).

69. Thirion, B., et al. Inverse retinotopy: Inferring the visual content of images from brain activation patterns. NeuroImage 33, 1104-1116 (2006).

70. Wammes, J. D., Norman, K. A., \& Turk-Browne, N. B. Increasing stimulus similarity drives nonmonotonic representational change in hippocampus. eLife 11, e68344 (2022).

71. Palan, S., \& Schitter, C. Prolific.ac-A subject pool for online experiments. Journal of Behavioral and Experimental Finance 17, 2227 (2018).

72. Brysbaert, M., Warriner, A. B., \& Kuperman, V. Concreteness ratings for 40 thousand generally known English word lemmas. Behavior Research Methods 46, 904-911 (2014).

73. He, K., Zhang, X., Ren, S., \& Sun, J. Deep residual learning for image recognition. Proceedings of the IEEE Conference on Computer Vision and Pattern Recognition, 770778 (2016).

74. Engstrom, L., et al. Adversarial robustness as a prior for learned representations. arXiv:1906.00945 (2019).

75. Madry, A., Makelov, A., Schmidt, L., Tsipras, D., \& Vladu, A. Towards deep learning models resistant to adversarial attacks. arXiv:1706.06083 (2017).

76. Ledoit, O., \& Wolf, M. Honey, I shrunk the sample covariance matrix. Journal of Portfolio Management 30, 110-119 (2004).

77. Kingma, D. P., \& Ba, J. Adam: A method for stochastic optimization. arXiv:1412.6980 (2014).

78. Loshchilov, I., \& Hutter, F. Decoupled weight decay regularization. arXiv:1711.05101 (2017).

79. Peirce, J. W. PsychoPy_psychophysics software in Python. Journal of Neuroscience Methods 162, 8-13 (2007).

80. Loper, E., \& Bird, S. NLTK: The natural language toolkit. arXiv:cs/0205028 (2002).

81. Caplette, L., Gosselin, F., \& West, G. L. Object expectations alter information use during visual recognition. Cognition 214, 104803 (2021). 
82. Holmes, A. P., Blair, R. C., Watson, J. D. G., \& Ford, I. Nonparametric analysis of statistic images from functional mapping experiments. Journal of Cerebral Blood Flow \& Metabolism 16, 7-22 (1996).

83. Hilton, J., Cammarata, N., Carter, S., Goh, G., \& Olah, C. Understanding RL Vision. Distill 5, e29 (2020).

84. Kriegeskorte, N., \& Mur, M. Inverse MDS: Inferring dissimilarity structure from multiple item arrangements. Frontiers in Psychology 3, 245 (2012).

85. Diedrichsen, J., Berlot, E., Mur, M., Schütt, H. H., \& Kriegeskorte, N. Comparing representational geometries using the unbiased distance correlation. arXiv:2007.02789 (2020).

86. Van der Maaten, L., \& Hinton, G. Visualizing data using t-SNE. Journal of Machine Learning Research 9, 2579-2605 (2008).

87. Charest, I., Kievit, R. A., Schmitz, T. W., Deca, D., \& Kriegeskorte, N. Unique semantic space in the brain of each beholder predicts perceived similarity. Proceedings of the National Academy of Sciences 111, 14565-14570 (2014).

88. Kim, G., Lewis-Peacock, J. A., Norman, K. A., \& Turk-Browne, N. B. Pruning of memories by context-based prediction error. Proceedings of the National Academy of Sciences 111, 8997-9002 (2014). 


\section{Acknowledgements}

We wish to thank Jeffrey Wammes for help with the initial image synthesis efforts and Frédéric Gosselin for helpful discussions about various aspects of the paradigm. We also thank our

funding sources: National Science Foundation and Fonds de Recherche du Québec - Nature et Technologies. The funders had no role in study design, data collection and analysis, decision to publish or preparation of the manuscript. 